452915328.

D5827. 5836 . 
$5-507$ 


$$
\begin{array}{r}
5.291 \\
328 \\
D 5827 \\
36
\end{array}
$$


$\left.\begin{array}{l}\text { Drabses } \\ \text { Hydrog, }\end{array}\right\}$ No. \& 2 , of

Sdg. Meh: $=2$ -

Turns

Cor'n
Date, Reel

Shot
Lead

Depth

i) 3

fms.

SdG. Cup

Amt. Bot'm

Character of
Bottom

Thermom. $\left\{\begin{array}{l}\text { Make } \\ \text { No. }\end{array}\right.$ Cor'n $\left\{\begin{array}{l}\text { Instr. } \\ \text { Stem. }\end{array}\right.$

Bottom Reading

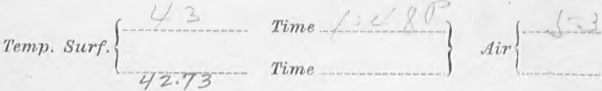

Density: Surf.

fims.

Wind: Direction

Force 2

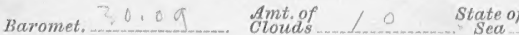

Weather ...........

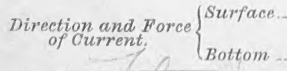

Tide

-1 Bottom

\section{General Locality}

\section{Position}

Chart used: No.

$\pi d d^{\prime} n$

Drift: Direction.

Distance

Depth of Haul

Apparatus and Rig used

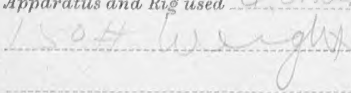

\section{Time towing}

h.

Remartes :

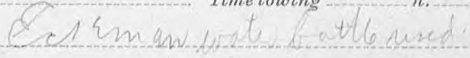


FMS, DREDGING CABLE.

M. Down. UP. M. $13800 / 4800$
FMS. J. DOWN. UP. M.

\begin{tabular}{l|l|l|l|l|}
\hline 0 & 3 \\
0 & 0
\end{tabular}

100

200

300

400

500

600

700

800

900

1000

1100

1200

1300

1400

1500

1600

1700

1800

1900

2000

2100

2200

2300

2400

2500

2600

2700

2800

2900

3000

8100

3200

3300

3400

3500

8600

3700

3800

3900

4000

4100

4200

4300

4400

4500

4600

4700

4800

4900 


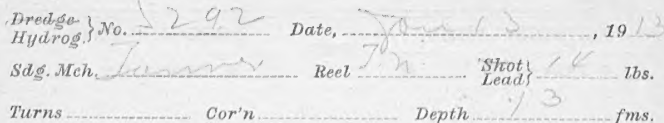

Sdg. Cup Amt. Bot'm

Character of Bottom

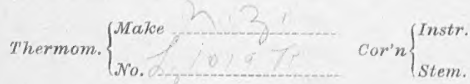

Bottom Reading $\backslash 2.71$. Cor'd Temp. 43.43

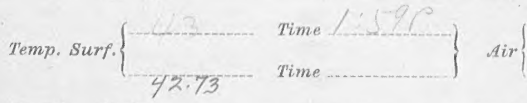

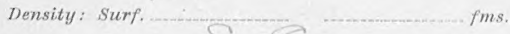

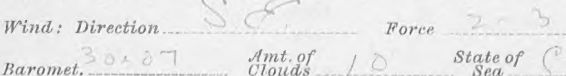

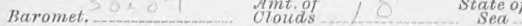

Weather

Direction and Force
of Ourrent. $\left\{\begin{array}{l}\text { Surface } \\ \text { Bottom }\end{array}\right.$

Tide

General Locality

\section{Position}

Chart used: No. Ed'n

Drift: Direction $)=$ Distance

Depth of Haut . Pr)

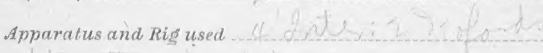

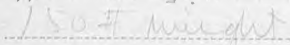

Remarks:

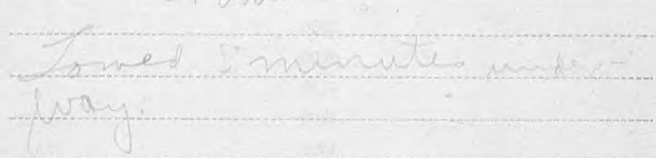


SOUNDING WIRE.

I... M. Down. UP. I M.
DREDGING CABLE.

FMS. M. DOWN. UP. M.

$0 / 153002 / 0800$

100

200

300

400

500

600

700

800

900

1000

1100

1200

1300

1400

1500

1600

1700

1800

1900

2000

2100

2200

2300

2400

2500

2600

2700

2800

2900

3000

3100

3200

3800

3400

3500

3600

3700

3800

3900

4000

4100

4200

4300

4400

4500

4600

4700

4800

4900 
$\left.\begin{array}{l}\text { Dredge } \\ \text { Hydrog }\end{array}\right\}$ No. S 293

Sdg. Mch.

Turns

Cor'n
Date,

Reel

SdG. Cup

Amt. Bot'm

\section{Character of}

\section{Bottom}

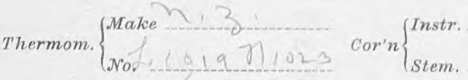

Bottom Reading 2.4L 4 Cor'd Temp. 43.93

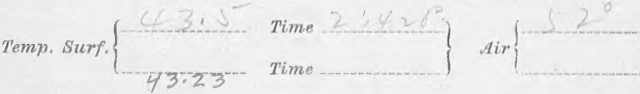

Density : Surf.

Wind: Direction

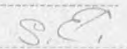

Baromet. 30,07 Ant. of

Force

fims.

Weather tbs.

Depth 7 fms.

. 
SOUNDING WIRE.

M. Down. UP. M. 236.0072000

FMS.

DREDGING CABLE.

C. Mown. Up. D. M.

\begin{tabular}{|r|r|r|r|r|r|r}
\hline 0 & 2 & 31 & 90 & 2 & 44 & 30 \\
\hline 100 & & & & & & \\
\hline 200 & & & & & & \\
\hline 300 & & & & & & \\
\hline 400 & & & & & \\
\hline 500 & & & & & \\
\hline 600 & & & & & \\
\hline 700 & & & & & \\
\hline 800 & & & & & \\
\hline 900 & & & & & \\
\hline 1000 & & & & & \\
\hline 1100 & & & & \\
\hline
\end{tabular}

1100

1200

1300

1400

1500

1600

1700

1800

1900

2000

2100

2200

2300

2400

2500

2600

2700

2800

2900

3000

3100

3200

3300

3400

3500

3600

3700

3800

3900

4000

4100

4200

4300

4400

4500

4600

4700

4800

4900 

$\left.\begin{array}{l}\text { Dredge } \\ \text { Hydrog }\end{array}\right\}$ No. $\frac{1}{2}<1$

Sdg. Mch.

Turns

Cor'n
Date, Reel

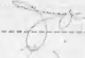

D. Shot

Depth

Sag. Cup

Amt, Bot'm

Character of

Bottom

Thermom. $\left\{\begin{array}{l}\text { Make } \\ \text { No. }\end{array}\right.$ Cor'n $\left\{\begin{array}{l}\text { Instr. } \\ \text { Stem. }\end{array}\right.$

Bottom Reading 449 . 8 Cor'd Temp. 4.4.8.8

Temp. Surf. $\left\{\begin{array}{ll}44,1 & \text { Time } 31,180 \\ 43.83 & \text { Time }\end{array}\right\}$

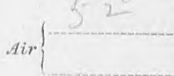

Density: Surf

fims.

Wind: Direction. S S ?

Force

Baromet. $\sum 4,63$ Amt.of / Clouds state of

Weather

Direction and Force
of Current.

Tide

General Locality

\section{Position}

Chart used: No

$E d l^{\prime} n$

Drift: Direction.

Distance

Depth of Haul.

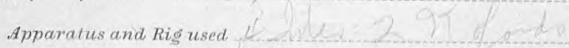

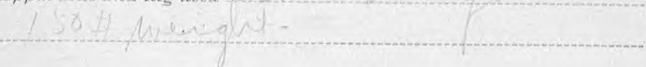

Time towing

h. $m$.

Remarks:
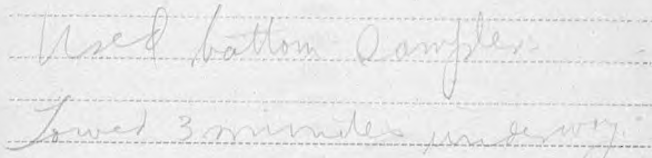
GOLNDING T WIRE:

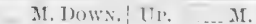
$5106 \mid 0 \% 3118100$
FMS | DREDGING (ABHE

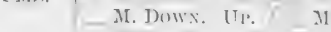

111)

$0.1316|013| 220$

200

:3in)

.100

500

(i)o

700

silo

906

1000

1100

1200

1800

$1 .+(90)$

1500

16006

1704

1800

1940

2000

2100

:2:204)

:2:300

2100

$\therefore 50$

$\because(0) 0$

2700

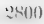

2900

$: 3000$

3100

$\because 100$

3:100

: 100

Bnto

$\because 6000$

:

조어세

:3900

f 4000

.1100

4900

म:301

1100

1,000

. $16(10)$

1700)

1500

(1900) 


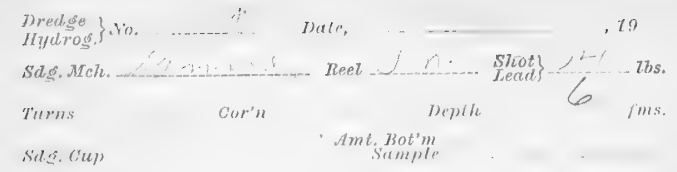

Charieter of

Thermom. $\left\{\begin{array}{l}\text { Make: } \quad \ldots \\ \text { No. }\end{array}\right.$

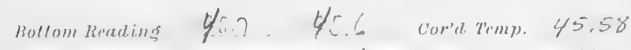

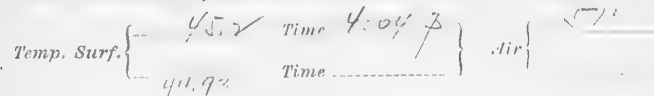

Density: Surf.

fms.

Wind: Direction

Foree

Buromet. 20 C Amtiof / a state of (?

Wrather

7) ireclion and Force
of Current. $\left\{\begin{array}{l}\text { Surter } \\ \text { Botlom }\end{array}\right.$

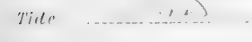

-...

Generel Lorality

Position

Chart used : No.

Ed'”

Drift: Direction Distenese

Deptir of IIaul ... In.

Apparatus and Rig used $L(\ldots$, , , , , ,

, ..... .

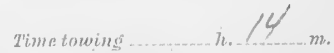

Remarlis:

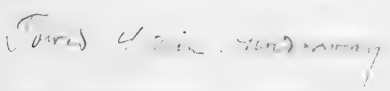


11-1023

M. Down. $\frac{U_{1}, \ldots . . .}{U^{\prime},{ }^{\prime}}$

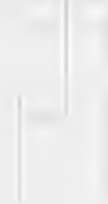

FMS.

1)RLID) IING CABLE

1010

200

:300

100)

500

(i)

\%oi)

800

90()

1000

11(1)

$1: 20()$

130)

1. $1(10)$

1500

$1(i)()$

1700

1 s.10

1900

?(100)

3100

203011

:3:30)

$\because 100$

2000

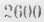

$2 \%(0)$

s⿻日木

s9u0

:3000

$\$ 100$

$: 3200$

:3.100

3800

:3500

$\therefore(0)$

$\because 70 *$

: $\$ 00$

:3:00

$.1000)$

.1100

$+200$

$4: 800$

4.400

1500)

4600

4700

1800

1900
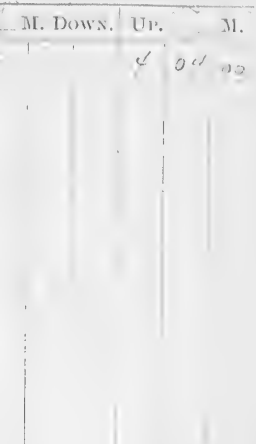

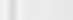




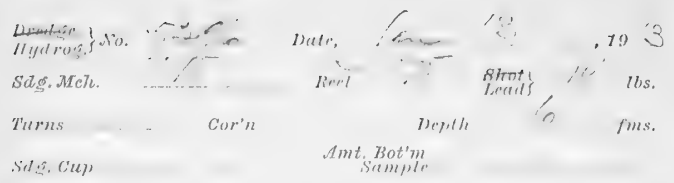
('hurreter or'
Butlom

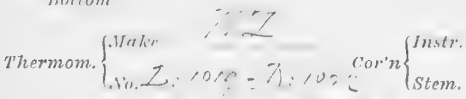

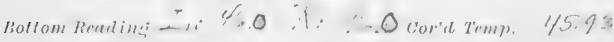

Temp. Surf. $\left\{\begin{array}{r}4 \sqrt{5} 8 \\ 775,3\end{array}\right.$

Time $4: 35-2$
Time

$\int \sqrt{1}$

Inrnsit!l: Surf.

lims.

Wind: Direction

$\$ S$

Force 4

Baromet. So. ol

Amt, of

10

State of

Weather 0

Direction and Fores
of Current. $\left\{\begin{array}{l}\text { Surfuer } \\ \text { Juttom }\end{array}\right.$

Tirle

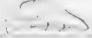

Cinemel Lorrtit!y

I'osilion

Chutrt usert: No. $F d^{\prime} n$

Hrifl: Hireetion Disian.m.

Depthe of Haut .....

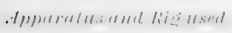

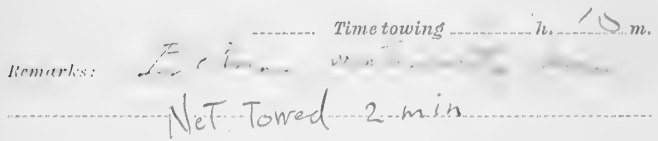

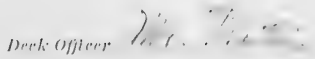

lixturaler

11 Heस: 
SOUNDING WIRE.

$\zeta^{5}$ M. DOWN. Ur. $\theta^{0} \mathrm{M}$.

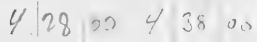

Iisti.

DREDGING CABLIE.

IT. Down. UT. M.

0

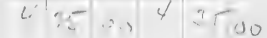

100

200

:30)

100)

i) 00

bici)

70()

800

(9)(0)

1000

110()

12()$(1)$

$1 \$ 3(0)$

1.100

1500

$1(i) 0$

$1 \% 00$

1800

1000

$\because 000$

$2100)$

2000

$2: 300$

$? 100$

2,500

2000

$2 \div 00$

$2 \mathrm{~S} 00$

29000

$30(10)$

$\$ 100$

$2: 3 x$

$: \cdots(10)$

: (1)

:30)

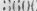

:ละก(1)

:

:क्षा)

.10(0)

.1100

4? 311

1:301)

1100

livo)

1400

1700

1800

.1900 


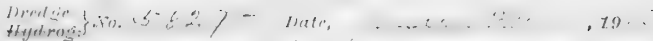

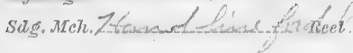

Turns

Cor'n

Depiti $12-12 \frac{1}{2}=6 \%$ ivins.

Sas. Cup

Aint. 73ot'm

Character of

Triltom

Thermom. $\left\{\begin{array}{l}\text { wakn } \\ \text { gis. }\end{array}\right.$

cornn $\left\{\begin{array}{l}\text { Instr. } \\ \text { stem. }\end{array}\right.$

liotlom limatims

Cor'd. Trmp.

Temp. Surp. $\left\{\begin{array}{l}4 \% \\ \ldots\end{array}\right.$

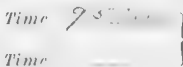

$\operatorname{lin} \mid=0$

Densit!l: surr:

fins.

Wint: Direction Whitam

Foree $0-1$

litromel. $30,4,-$

Ami.of

sicutrof

Wrenther - -

Tirection and Foree
of Current. $\left\{\begin{array}{l}\text { Surface } \\ \text { liallumi }\end{array}\right.$

Tille

Grneral Loratily

Posilion

Ghest usert: . is.

E.l'n

Drift: Direction

Distance.

Depth of naut. botine

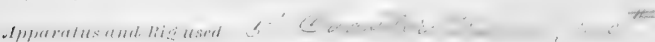
$, \because, \ldots, \quad \because, \therefore, \ldots, \ldots$

Timeturins

n. $1 \%$

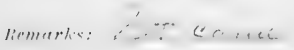


SOUNDING WVIRI:

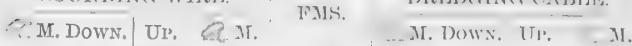

DIREAING (CABTAL

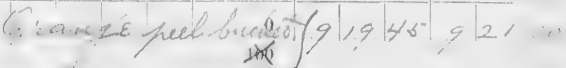

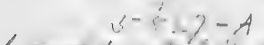

शेषार

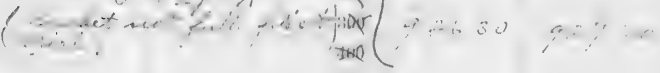

.

(600)

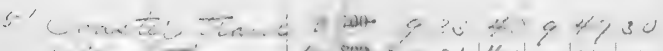

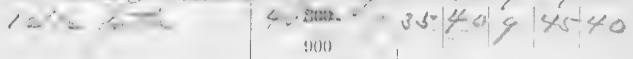
1000

1100

$1:(0)$

1800

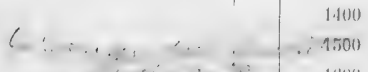

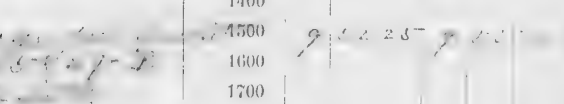

i X.

1)(

1900

$: 00(0)$

2100

2001

2300

स्4(10)

:2500)

$\because(6)$

ะสาก

$3 \times(1)$

2900

$: 0000$

3100

:":on

(3)

3400

:3900

:150)

:

:3500

$3: 100$

.100\%

410!

4200

4:301

4400

4.00

$460 \%$

4700

$150(0)$

$.19(4)$ 


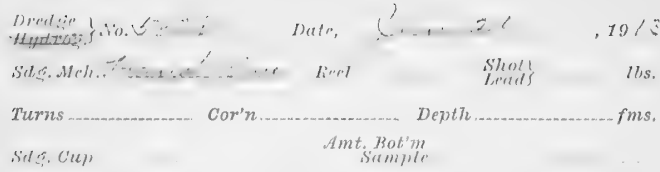

Character of Boltom

Thermom $\left\{\begin{array}{l}\text { wreler } \\ \text { Noo. }\end{array}\right.$

$$
\text { Cor'n }\left\{\begin{array}{l}
\text { Instr. } \\
\text { Stem. }
\end{array}\right.
$$

\section{Dollom Reralins}

Cor'd Trmp.

Temp. Surf. $\left\{\begin{array}{l}4: \infty \\ \end{array}\right.$

Tim" $/ y^{\prime}, \cdot$

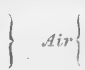

Time

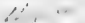

Drisity: Surf:

fins.

Wind: Direction... Vesternces.

Force $0-1$

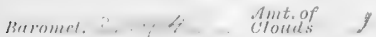

State of

Weather Nege

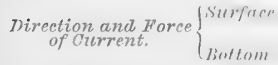

Tide $\ldots t^{t}$

General lomtity

\section{Position}

Chart used: No, Itd'n

Drift: Direction

Distanec

Depth of IIaut - S. Theree.

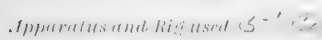

Jicmurlis: 


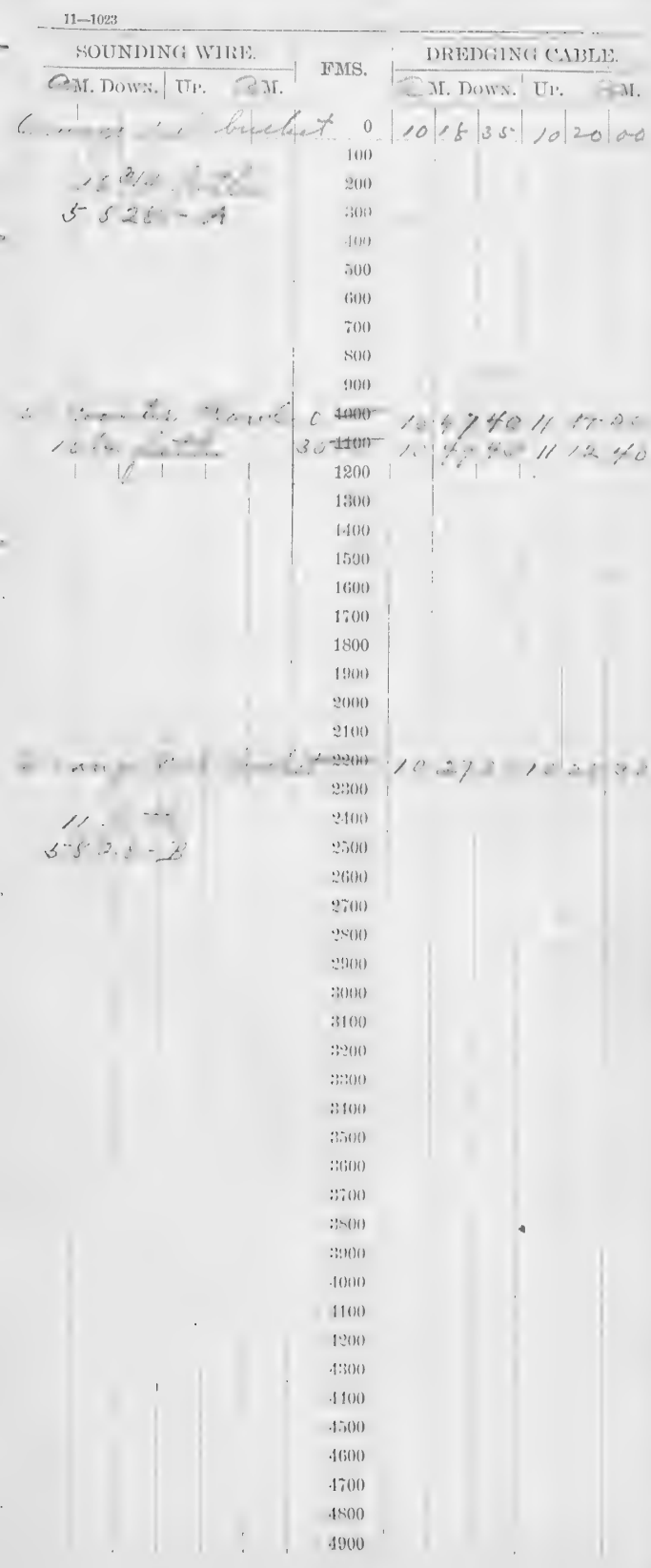




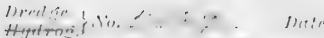

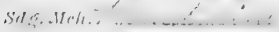

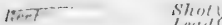

$1 !$

Turns

Cor'n

Dejith

llis.

Sid $6 .(1)$

Amt. B3ot'm

Character of

Bollonit

Thormom. $\left\{\begin{array}{l}\text { Make } \\ \text { No. }\end{array}\right.$

$$
\text { Cor'n }\left\{\begin{array}{l}
\text { Instr. } \\
\text { Stem. }
\end{array}\right.
$$

Bottom Reading Cor'd Temp. Temp. Surf. $\left\{\begin{array}{l}S^{\circ}-0^{\circ} \\ \text { Time }\end{array}\right\}$ Air $\}$ fins.

Density: Surf

Q $=i$

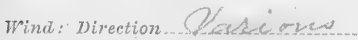

liredmet. 30 itan Amt of State of

Wenthw.

Direction and Force
of Current. $\left\{\begin{array}{l}\text { Surites } \\ \text { Jinfom }\end{array}\right.$

Tide...chle

Ginemel hourtil!

Position

rible Hat: Ais.

EI'

frifl: Hiration Hisfander

nepth of Haul

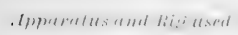

- $\therefore$ inti,

time tumins

ii. $/ \mathrm{m}$.

liemmer 
SOUNDINA WERT.

M. DOWN. UP. .... M.

Fis.

DTREDTING (

$13-5+2=-4$

(i)

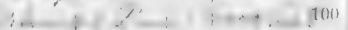

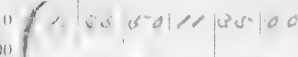

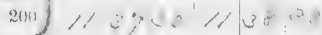

$\because$ :11

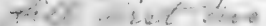

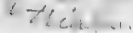

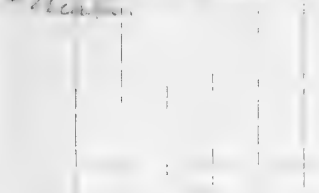

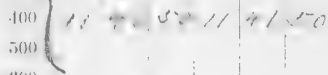

ti(n)

700

siot

9(30)

1000

1100

1200

$(4-3 \therefore, \therefore) \div$

II $44,8,16 \cdot 3,5$

$30+100$

1500

(60)0

1700

1800

1900

2000

2100

2200

2300

260

2500

2000

2)

खs(0)

2900

$: 3000$

3100

$: 3: 300$

3300

3400

300

;)(600

3700

3800

:3900

4000

$\$ 100$

4200

1300

.1100

.1500

4500

.1700

1800

.1900 


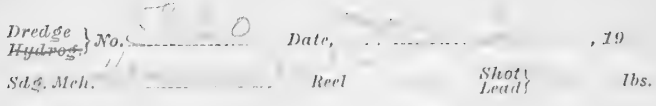

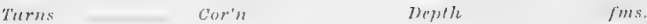

Sils. ('up) Amt, Bot'm

Character of

Bottom

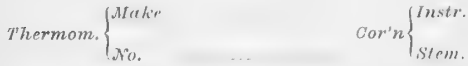

Butiom Reraling

Gor'd Trmip.

Temp. Surf. \{

Time

Time. $\{. i r$

Densily: Surf.

Wind: yirection. Conf

jim.s.

Forre

Baromet. 30,41 Amt of

Sicitiof

Weather

Direction and Force $\left\{\begin{array}{l}\text { Sulwitur } \\ \text { of Current. }\end{array}\right.$

Ticle

General Torality

\section{Pusiliun}

chart uspd: Mo.

I)rift: Jivertion

Depth of Haul

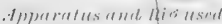

$k l^{\prime} n$

Dislaree

Time turvint?

h.

III.

limark: 


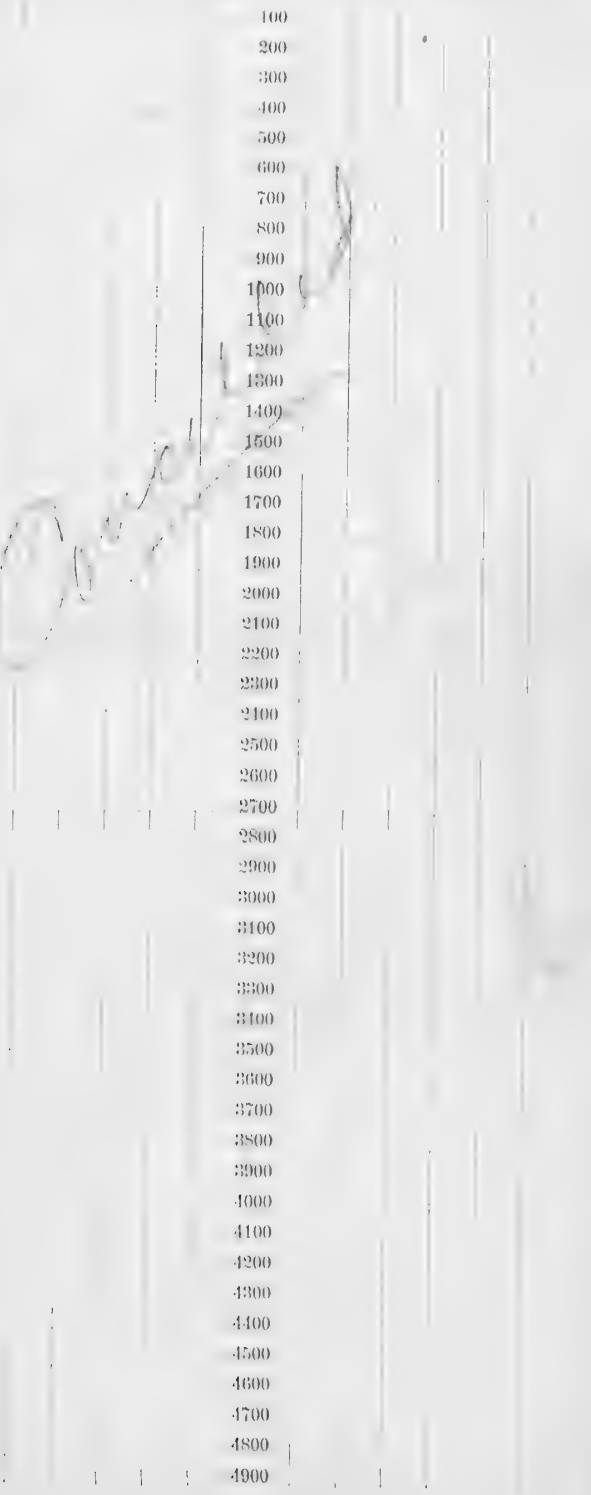




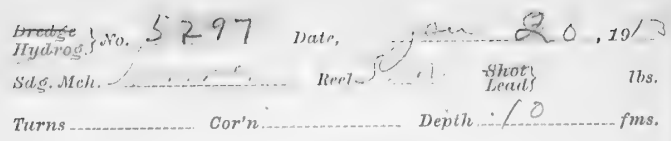

\section{Sa. Cup}

Amt. B3ot'm

Character of

Thermom. $\left\{\begin{array}{l}\text { Make } \\ \text { No, } 1019\end{array}\right.$

Bottom Readings $\therefore$, C... Cor'd Trmp. $47 \% 3$

Temp. Surf. $\left\{\begin{array}{ll}4 / 8 & \text { Time } / 930, \\ \text { Time }\end{array}\right\}$ Air.

$$
47.73
$$

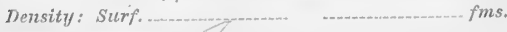

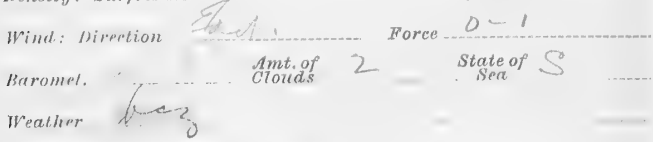

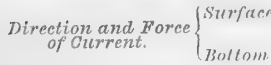

Tille

General Locality

\section{Position}

Chart used: No,

Eid'n

Drift: Direction Ceucheren d. Distame

Depth of maut Sutc.

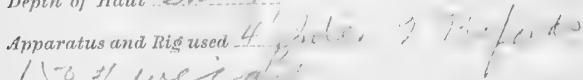
$11-0=1$ bue i

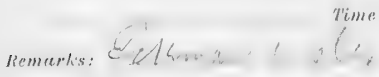

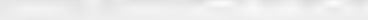


SOUNDING IVIRE

P M. Down. Un?

FMts.

1)RIEDGING CATBLI\%.

$12|5 / 001| 0120|\ldots 0 / 2| 16|0| / \mid 010$

160

200

$30(1)$

1110

.500

(ii)

$\approx 00$

$S^{\prime}(1)$

900

1000

1100

1200

1300

1400

1500

1600

1700

1800

1900

$29(00)$

2100

$\because 200$

$2: 301$

$\because 400$

2500

$\because(6) 0$

2700

2800

2900

$: 3000$

3100

$: 200$

:3:100

3100

3isin)

$\because 3600$

3001

:3ห(1)

:3901

1000

4100

1:00)

4300

1.100)

1.60)

Hian)

1700

4800

4900 


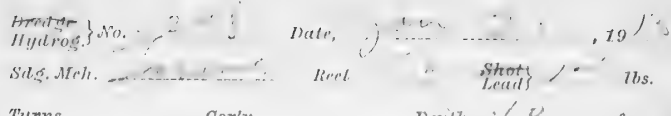

Turns

Cor'n

Depth $:(2)$ fms.

Sils ('up

Amt. Bot'm

Character of

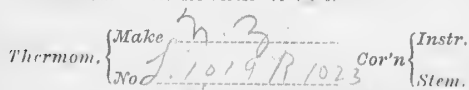

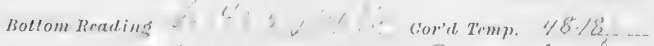

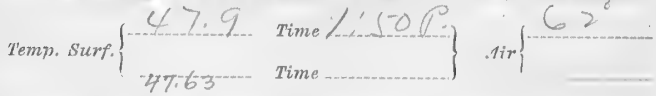

fms.

Innsity: SnrF.

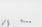

Wind : Jirention

Forer

zaromet. 30,38

Wealler w

Direction and Foree $\left\{\begin{array}{l}\text { Siufise } \\ \text { of Current. }\end{array}\right.$

Tide

\section{Cienerel loretity}

\section{Position}

Chart used: No. $\pi u^{\prime} n$

Drift: Directiont $\hat{k}, \ldots(\ldots, \ldots+$ Distumee

Depth of Haut

Apmaratus and kisused l t. $\because, \ldots$, ,

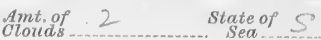

i

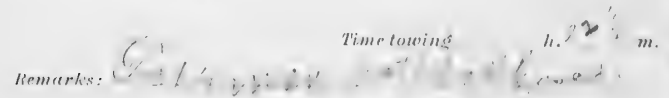
$11, \ldots+\ldots+\ldots$ 


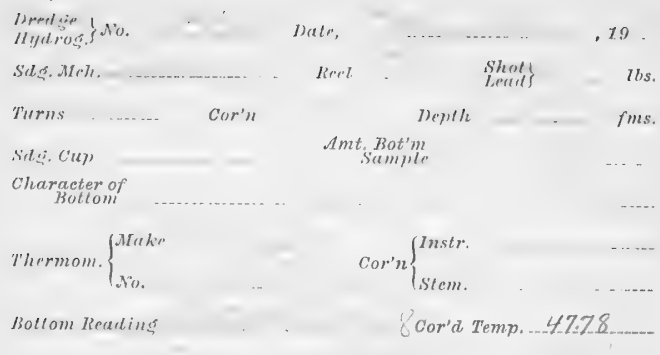

Trmp). Surf.

$\because \% r_{2}$

Irnsityl: surf.

INind: Jirention

Baromet. 30.38

reather

Direction and Force
of Current. $\left\{\begin{array}{l}\text { Surfase } \\ \text { Dinltom }\end{array}\right.$

Tille

Time

Iime

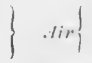

fims.

Foree

Amt.of 2 State of 5

General boenlity

\section{Position}

Chart used: No.

$\ddot{I} d d^{\prime} n$

jrift: Direction

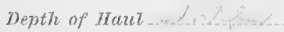

Apparatus and Rig used 4 ....

1 i. laser

Timeloutin:s

h.

$m$.

Remarks:!

$$
1,1+11
$$

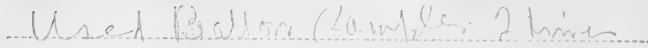


SOUNDING WIRE.

M. Dow. TH. M. M.

FMs.

DREDGING CABLE.

(2) Dows. U1. . II

100

200

300

100)

500

(6)

ร01)

800

!)(1)

1000

1100

1200

1800

1.400

1500

$16(0)$

1700

1500

1901)

20010

$\because 100$

20200

2:300

$\because 900$

2700

2600

2700

$\because 400$

2900

$: 3000$

$: 3100$

$: 300$

:3:300

3100

B3:00)

:1600

:ร7)(

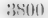

:3000

$.10011)$

1100

(1)(1)

.1:000

1.100

.1500

4000

4700

1800

1900 


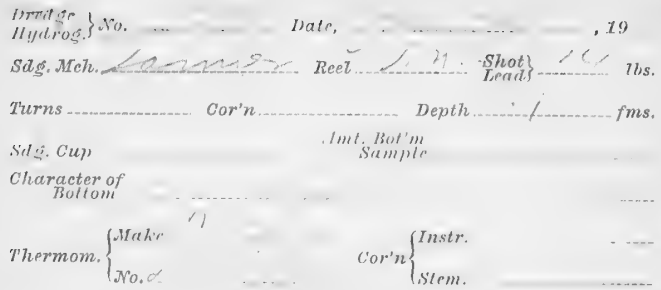

\section{P'osition}

(shart uswl.: Nin. Ed' $n$
11.ifl: Direction
Distunce

Depth of Haur ... Che

Apparatus and Rig used, 1, Ses?

I o.fl (uje catal) 
11-1023

SOUNDING WIRE.

M. Dow. IT. M.

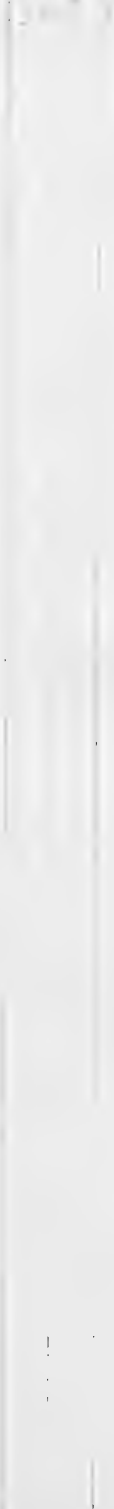

FMS.

DREDGING CABLE.

M.

200

:30)

.100

500

(ion)

$\tau 00$

800

900

1000

1100

1900

$1: 00$

1.100

15001

1600

1700

18(0)

1900

$\therefore(0)(1)$

2100

$2: 201$

2900

$\because-900$

2500

2800

$27(1)$

$2 \times(1) 0$

29900

$\therefore(0)$

3100

3200

(3)300

3100

3):00

:3io)

:

$: 3400$

3900

1000

1100

4200

4:300

1400

.5000

Itevo

. 1700

1800)

f(S) 


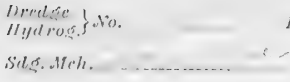

I) ale,

19

Turns

Cor'n

Reet

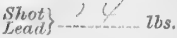

Sid $\{$. (tu)

sulmple

Character of Botlom

rhermont $\left\{\begin{array}{l}\text { Afuke } \\ \text { io. }\end{array}\right.$ Cor'n $\left\{\begin{array}{l}\text { Instr. } \\ \text { stem. }\end{array}\right.$

Botfom Ripading Cor'd T'm)

THm). Surf.

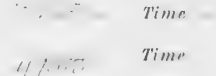

Density: Sturf.

$$
\{\operatorname{sir} 1
$$

ims.

Hnind: Hirntion

Forre

Virromel, $20, n, 8 \quad$ Amt of $\quad 1$ State of

Ifrather

Direction and Force
of Current. $\left\{\begin{array}{l}\text { Surface } \\ \text { Iitultom }\end{array}\right.$

Tille

Generel Loratily

Iosition

thart usul: No.

$F i n$

Drift: Direction!

$n ! j+2 \times+2 ;$

Distance.

Depth of Haul

Apparatus and Rig used

Time towin:s

h.

$m$.

Hemert: : 
SOUNDIN( + VIIRE.

M. Down. U Ur, … M.

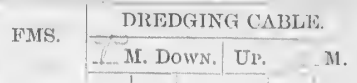

100

200

$\$ 00$

.100

500

(50)

ז00

s00

900

1000

1100

1:00

1300

1400

1500

I (50)

1700

1800

1900

2000

2100

2200

2300

24. 400

2500

2600

2700

2800

2900

3000

3100

:

:3:300

3400

3500

$: 300$

$3 \pi 00$

$\$ 3800$

:

.1000

4100

4200

.1300

4400

4500

4600

.700

1800

$4900)$

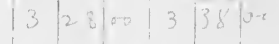

.




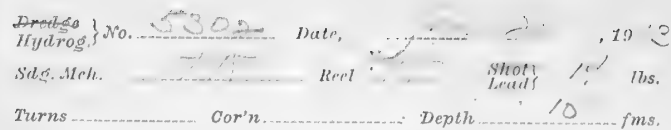

Sug. Oup _... Amt. Bot'm

Character of

liotiom

Thermom $\left\{\begin{array}{l}\text { Make } \\ \text { No. . Nol }=102.3\end{array}\right.$ Cor'n $\left\{\begin{array}{l}\text { Instr. } \\ \text { Stem. }\end{array}\right.$

Bollom Realins * : - ? Cor'd Trmp. 17.08

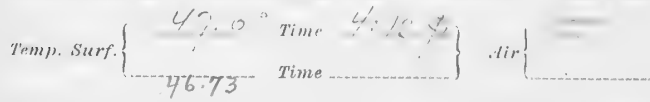

Density: Surf. fims.

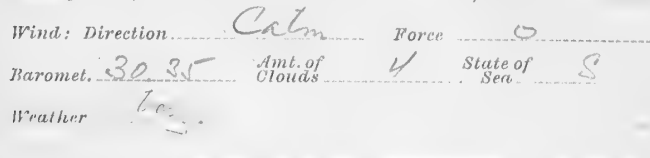

\begin{abstract}
Direction and Force $\begin{aligned} & \text { Surfact } \\ & \text { of Current. }\end{aligned}$
\end{abstract}
Tille

General loratily

Position

Chart used: No. E'din

Drift: Direction..... Ans Jures...... Distance

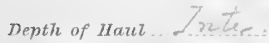

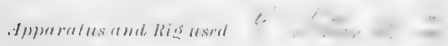

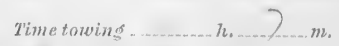

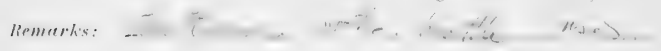

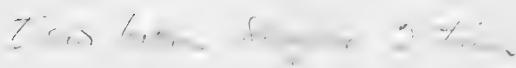




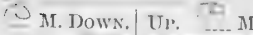

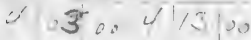

FMS. DREDGING CA13LI:

100

1)

405 60 4 y

200

$\because: 00$

.100

500

(i) 10

\%00

$\mathrm{s}(0)$

900

1000

1100

1:200

1300

1400

1500

$1(; 0)$

1700

1800

1000

2000

2100

2200

2300

2100

3500

2600

2700

2800

2900

$\$ 3000$

$\$ 100$

:2:00

33:300

3100

3500

$: 360$

:\%

3800

$\$ 3100$

4000

4100

4200

4:00

4400

4500

4600

.1700

1800

4900 


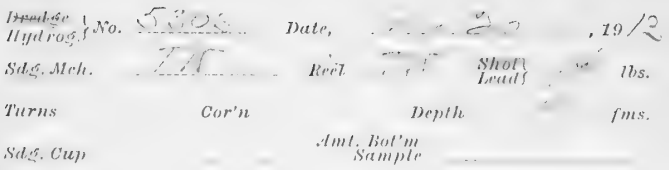

Character of
Bottom

$$
\text { Whrmom. }\left\{\begin{array} { l } 
{ \text { Hater } } \\
{ \text { No. } 1 0 1 9 \ldots - 1 0 2 3 }
\end{array} \text { Corn } \left\{\begin{array}{l}
\text { Instr. } \\
\text { Stem. }
\end{array}\right.\right.
$$

diatom Reading '

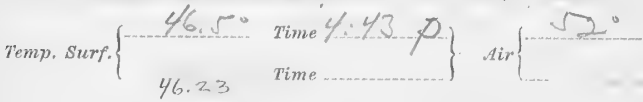

Density: Surf. fms.

Wind: Direction

Baronet, 30,35 Ami, of

Force

0

Weather

\%er

Direction and Force
of Current. $\left\{\begin{array}{l}\text { Surface" } \\ \text { Bottom, }\end{array}\right.$

Tide

General Loratily

Position

chart used: No. $E d d^{\prime}$

Drift: Direction Distance

Depth of Haul Tater

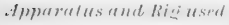

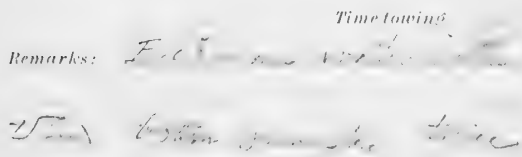

h. $\because m$.

Derek of leer.

11-10:3 
$11-1023$

SOUNDING WVIR.

U M. Down. Ur, W. M

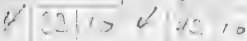

FMS. DIREDGING (AIBLE

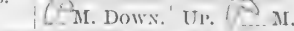

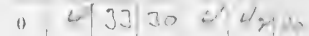

100

200

:390

400

500

(60)

700

800

900

1000

1100)

1200

1300

1400

1500

1600

1700

1800

1900

2000

2100

2200

2:300

2400

2500

2600

2700

2800

2900

3000

3100

:3200

: 3300

3100

3500

3600

: $\% 00$

3800

$: 1900$

4000

$\$ 1100$

4200

4300

1100

4500

4600

+ 700

4800

4000 


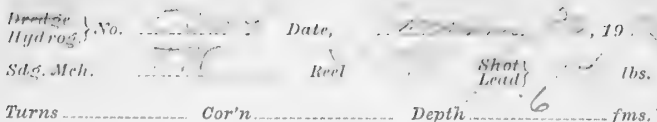

Turns

Cor'n Depth fms.

Sid 소. Cul

Amt. Bot'm

cheredeter of Bottom

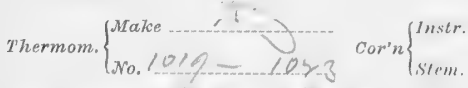

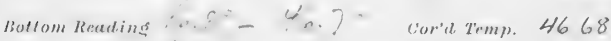

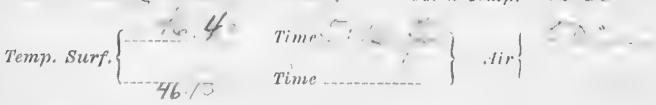

1)ensity: Surf.

fims.

Wind: Direction.... OR Forep

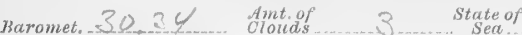

Weather ..........

Direction and Foree
of Ourrent. $\left\{\begin{array}{l}\text { Sur/iur } \\ \text { Boltom }\end{array}\right.$

Tille

Genmal Loratity

Posifion

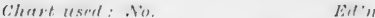

Drift: Direction..... Le

Depth of Haul ....... T2 The

Apparalus and Ris usal

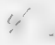

Timetorvin:

h. $\stackrel{1}{2} m$.

LPmurllt:s:

(1)

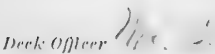


SOUNDING WIRL. $\because$ M. Down. UP. U. M. $\therefore 1021=0$
FMS

DIREDGING CABLE.

FMS. VMT. DOWN. Ur. QM.

200

:300

900

500

(i) (1)

ז00

800

900

1000

1100

1200

1300

1400

1500

I600)

1700

1800

1900

$\simeq 000$

$: 100$

2200

2:300

2400

$\div 500$

2000

$2 \% 00$

2900

2900

3000

3100

$: 3200$

33300

$3 \$ 00$

3500

$: 3600$

$\$ 300$

:3800

3900

4000

4100

4200

4300

$\$ 100$

.1500

1600

$47(4)$

4rivo

म!9)

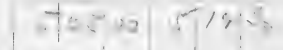

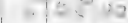

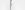

,

.

[




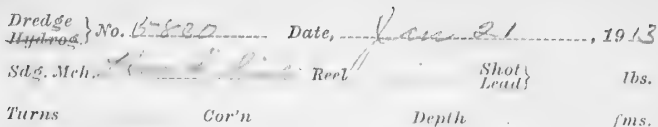

Sag. Cup ... Amt, Bot'm Sample

Character of Bottom

Thermom. $\left\{\begin{array}{l}\text { Mlake } \\ \text { No. }\end{array}\right.$ ('or'n $\left\{\begin{array}{l}\text { Instr. } \\ \text { Stem. }\end{array}\right.$

Boflom Readins

cor'de Trmp.

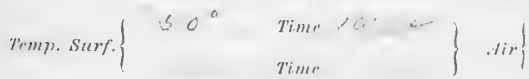

Hensity: Surf: fms.

Wind: Direction. Porce

Baromet. 30.26 Ant of 3 Stouds Sen of

Weather

Direction and Force
of Current. $\left\{\begin{array}{l}\text { Surfuce } \\ \text { Bu/tom }\end{array}\right.$

Tille - קs

General Locality

Position

Chull used: Are.

E'l' 'I

Drift: Direetion

Distance.

Depth of Haut fiton

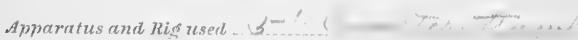

Timetwoins: H.

Remarks: 

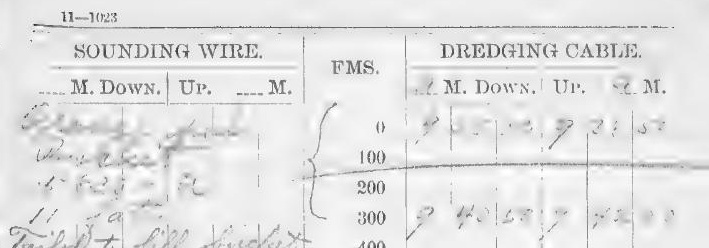

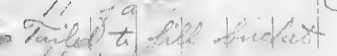

$21, w+7+\cdots+500$

.
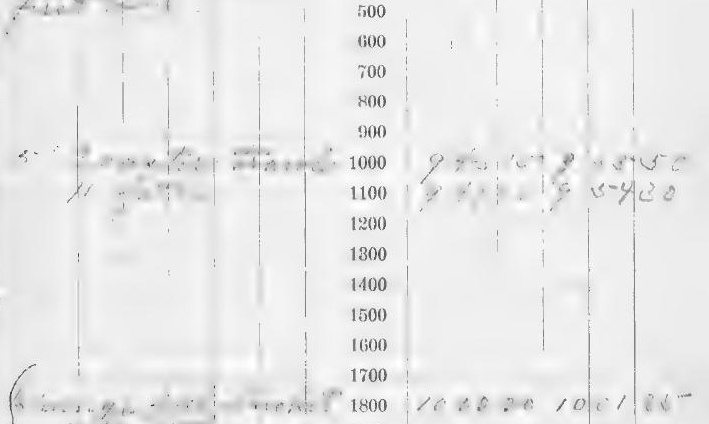

$\int$

$1800 / 6 \therefore 6: 0,0,1 / 26$

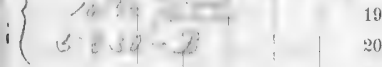

2000

2100

2200

2300

2400

2500

2600

2700

2800

2900

3000

3100

:3200

33300

3400

3500

3600

$\$ 700$

3800

$: 9000$

4000

4100

$4: 00$

4300

4400

4500

4600

4700

4800

$4900)$ 


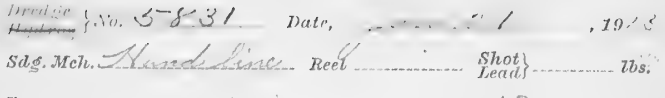

Thrns

Cor'n

Depth:_Lis $f m s$.

Sils: ('u\})

Amt, Bunt'm

Character of

Bottom.

Thermom. $\left\{\begin{array}{l}\text { Make } \\ \text { No. }\end{array} \quad\right.$ (ior) $\left\{\begin{array}{l}\text { Instr. } \\ \text { slem. }\end{array}\right.$

lioflom. Reatins _. Cor'de Timp.

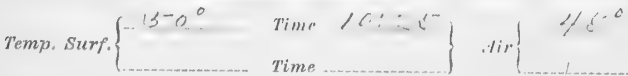

1)ensily: Surf.

ins.

Wind: Direction ... Toree ...

Baromet. $3_{2} 2, \quad$ Amt.of 3 State of

Weather

Direction and Force
of Current. $\left\{\begin{array}{l}\text { Surficer } \\ \text { Bottom }\end{array}\right.$

Tirle.

(iv)erel lowalily

Insition.

Chart usert: No.

Ed, $n$

Drift: Direction

Distance.

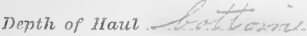

Apparatus and lite used

Nemertis: 
$-11-11: 23$

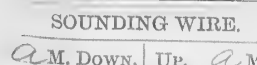

FMS.

DREDGING CABLE.

LM. Down. UP. A. M.

A Mr. Down. Ur. Q M.

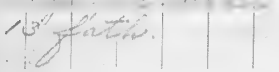

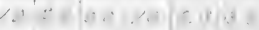

100

200

:301!

(190)

iso()

(i)i)

พิ1)(

800

(1)(1)

1000

1100

1200

1300

1.100

1500

$1(0) 0$

1700

1si()

1901)

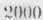

2100

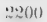

2:300

2) 100

2500

$\therefore(60)$

2700

प्रे(1)

?3:19)

:3)(1)

3100

:3:200

:3:3011

$: 3100$

:2:00

:460)

$3 \pi 00$

:3(0)

$: 3000$

f(0)10

1100

19() (1)

1.300

1400

(4)

f(1)10

17(1)

14011)

f(90) 


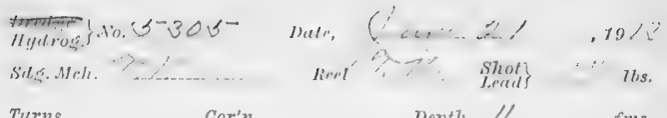

Turns

Cor'n

Depth...// fins.

Sils, ('u)

Amt. Bot'm

Character of

Bollom

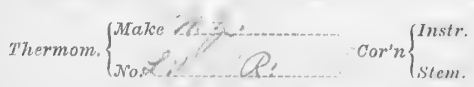

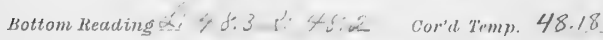

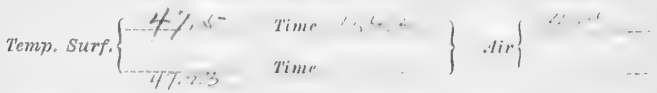

lensity: surf:

fins.

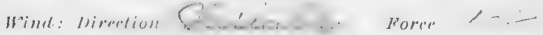

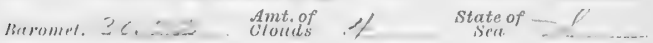

Weather anter.

Direrlion and Forec $\left\{\begin{array}{l}\text { Sinfirer } \\ \text { of durrent. } \\ \text { Bottom }\end{array}\right.$

ind 1

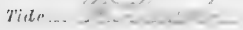

\section{Generel Lorelily}

Iosition

Cleart used: No,

$\pi d ' n$

Hrift: Direntimn

Disturue

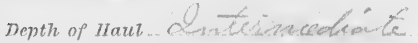

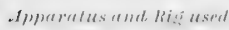

1.

Time towins

$72,+2 \ldots m$

Remarles:

$\therefore \therefore$ 

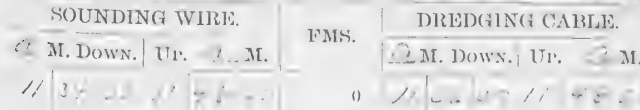

(1)

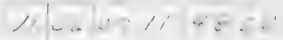

100

200

300

400

500

(60)

\% 00

800

(900)

1000

1100

1200

1300

1400

1500

I (i00)

1700

1800

19)0

?000

2100

2000

2300

$\because 100$

25000

2000

:37 700

2800

me(u)

:3000

$\$ 100$

:2000

:":.:(1)0

B(0)

300

:3tion

3700

issol

:2000

10)1010

11110)

$f(0)()$

1:300)

1)(0)

1500)

46:00

1700

1800

4900 


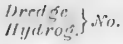

Sdes. Heth.

Turns

Sillê. Cup

Character of Boltom

thermom. $\left\{\begin{array}{l}\text { Mrelie } \\ \text { Jis. }\end{array}\right.$ Cor'n $\left\{\begin{array}{l}\text { Instr. } \\ \text { stem. }\end{array}\right.$

Jollom Rerdins Cor'a trmp. 47.7.8

Trmen. Surf. \{

$$
-47.73
$$

Time

Time 1)ate,

Rinel $6)$ Depth

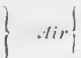

, 10

lbs.

1)rusityl: Surf.

"Mind: Jirretion

an oth fins.

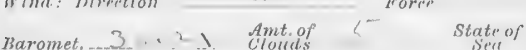

Weather .... bean

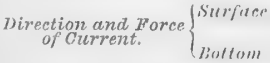

Tide

General Lorality

\section{Position}

Chart used: No.

$x d ' n$

Drift: Direction

Distance.

Depth of Haut

Apparatus and kig used.

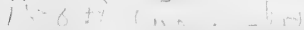

Time towing 7. . 10.

Remarks:

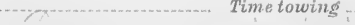

fims. 


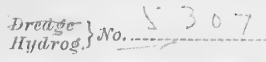

sas. Heh.

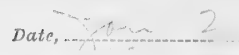

\section{Turns}

Cor'n

Row

Shot

liss.

Sils. Ciu

lejulh

fms.

Amt, T3ot'm

Sitmple.

Character of

Toltom

I'hermom. $\left\{\begin{array}{l}\text { Make } \\ \text { No. }\end{array}\right.$ Cor'n $\left\{\begin{array}{l}\text { Instr } \\ \text { Sistem. }\end{array}\right.$

Bottom Reading Cord Trmp, 4/7. ח $2 \ldots$

Temp. Surf. $\left\{\begin{array}{l}\text { Time } \\ \text { Time }\end{array}\right\} \operatorname{Air}\{\ldots$

Density: Surf. fms.

Wind: Direction.... Force ...

Baromet. 30,17 Ant. of State of

Weather $12 n$

Direction and Foree $\{$ surfare of Current. $\{$ Bultum

Tille

\section{Generat Joortily}

\section{prosition}

Chart usedt: . Vis. Eil'n

Drift: Direction

Distance.

Depth of Haul . t t t

Apparatus and Ri@ resed

Remurlis: 
SOUNDING WIRE.

MI. DOWN. TI: _...
D)IREDG $*$ ING C $\triangle$ B LLI:.

TMS.

MI. DUWN.I UT.

M.

(1)

100

200

3() 0

.110

500

(i)0

\%()

$8(1)$

(1)()

1000

110 )

12(9)

1300

1.100)

1500

1600

1700

1800

1900

2000

ज्ञात)

:2:200

:2:30)

$\because 100$

2500

$26(0)$

2700

ख्या10

$29(2)$

$:(100)$

3100

$\$ 200$

:3:300

:3100

8500

$: 300$

$: 300$

: $\mathrm{s}(0)$

$: 390)$

(1) $(1)$

1100

1200

4300

1400

$.1500)$

. 1600

.1700

(स⿻)

1900 


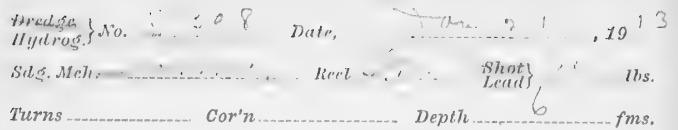

Sag. Cup

Amt. Bot'm

Character of Bollom

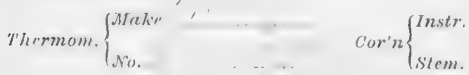

Botlom. Readins Cor'd Temp. $46, \$$

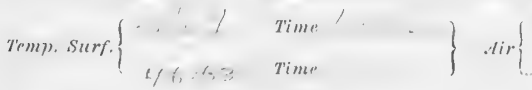

Density: Surf. fms.

wind: pirection

Buromet. : 3,13 Amt of 4 State of. 8

Weather l....

Direction and Force
of Ourrent. $\left\{\begin{array}{l}\text { Surface } \\ \text { Bottom }\end{array}\right.$

Tide

\section{General Lomality}

\section{Prsition}

Chart used: No. $I d^{\prime} n$

nrift: nirertion then n. no bे.

Distanes

I) inth of Iluul

Apparalus and Ris used'

Remarks: 


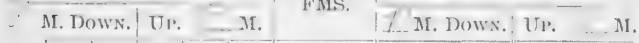

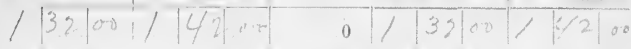
10)

20()

$\$ 300$

(1)0

500

(i) ()

\%00)

(5)

()()

1000

110 ()

$12(10)$

1301

1400

1500

$1(00)$

1700

1800

1900

2000

2100

2006

$2: 300$

$\because 100$

4500

2600

$2 \% 00$

2800

:ख)(9)

:3000

$: 3100$

:2:00

:3:300

$: 31(n)$

2300

$3(300)$

:3700

:35011

:अ)(

. $(0001)$

1100

1201

(1:30)

1100

$1500)$

14:00

(a)

.1800

4000 


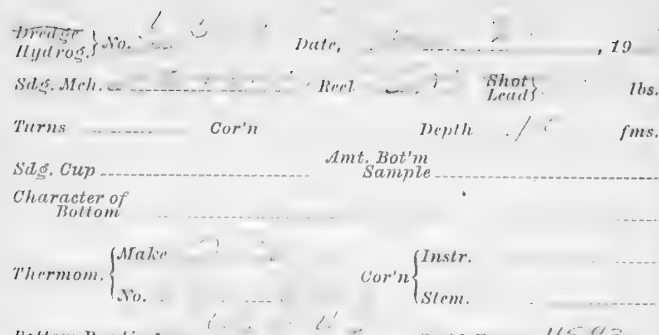

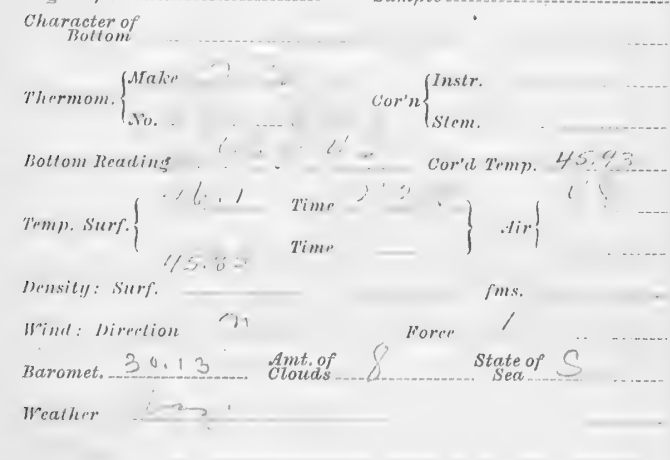

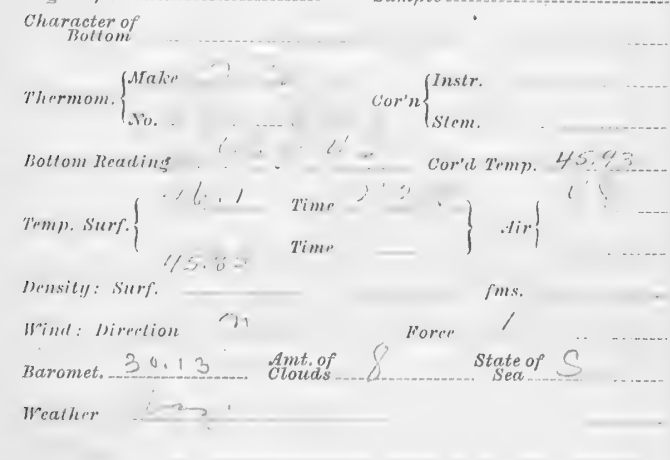

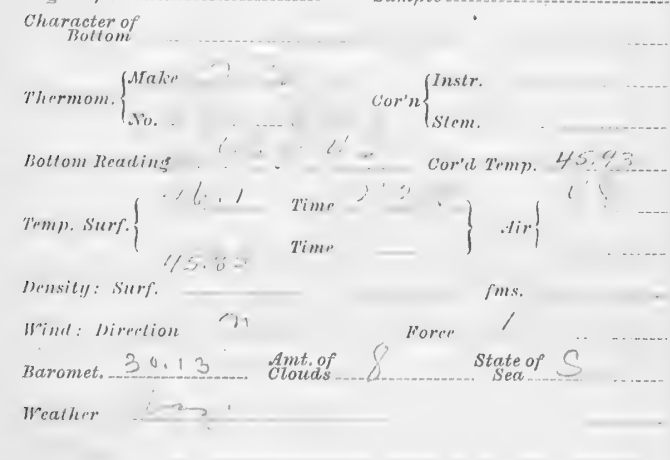

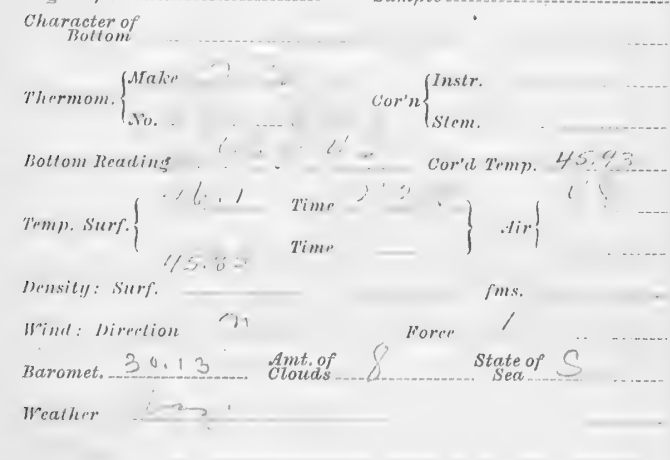

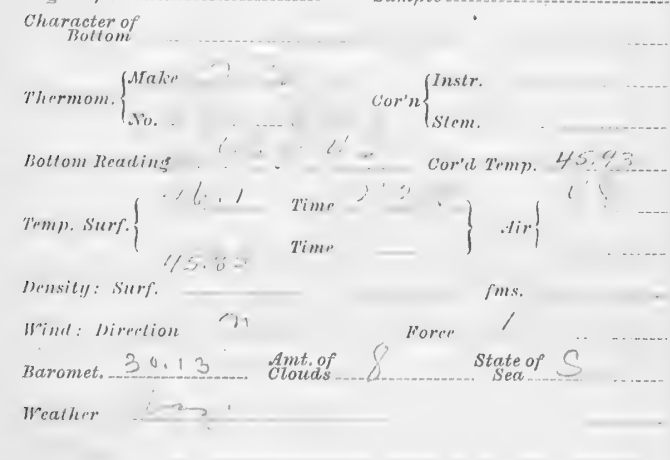

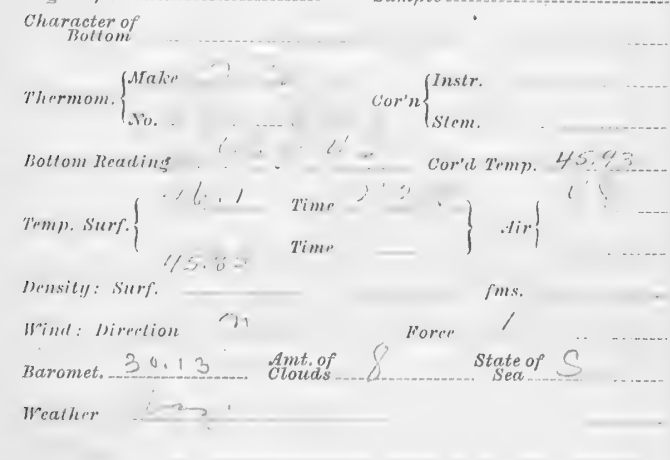

Direction and Force
of Current. $\left\{\begin{array}{l}\text { Surfurn } \\ \text { Butfom }\end{array}\right.$

Tirle

General Loerelity

Pasition

Chart used: No.

$E\left(l^{\prime} n\right.$

Drift: Direction. Distance

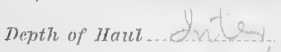

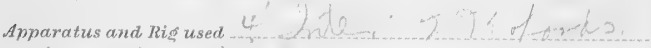

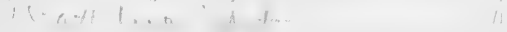

fms. 
SOUNDING WIRE.

M. DOWN. UP. M.

$21090012 \sqrt{400}$
DREDGING CAILE

INMS.

M. Down. UT. M.

210710012117100

200

300

100

500

(60)

700

800

900

10() 0

1100

$1: 200$

1300

1400

1500

1600

1700

1800

1000

2000

2100

2200

2300

2100

2500

2600

$2 \% 00$

$\approx 800$

2900

$\$ 3000$

3100

$3: 00$

$3: 300$

3100

3500

3600

$: 3700$

3800

3900

4000

4100

4200

4300

4.400

1500

4600

4700

4800

.1000 


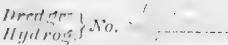

7)ati,

, $19 / 1$

Sd s. Heh......

IRerl

lis.

\section{Titrus}

Cor'n

Sils. Citu

Amt, Bot'm

Eharacter of

fhermom. $\left\{\begin{array}{l}\text { Maker , } \\ \text { No. }\end{array} \quad\right.$ ror'n $\left\{\begin{array}{l}\text { Instr. } \\ \text { stom. }\end{array}\right.$

Bollom Readins

cord Trmp. + 5,83

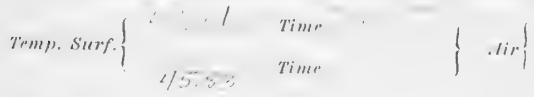

Jensity: Surf.

fins.

Wind: Direction

3aromet. $3,0,12$

Aint. of

$\theta$

clolids

Force

II callerr

Direction and Force
of Current. $\left\{\begin{array}{l}\text { Surfare } \\ \text { Sot(o)!I }\end{array}\right.$

Tithe

Ginnerl Lofalily

Position

Chart used: No.

Kd'n

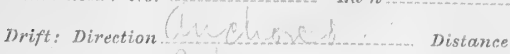

Deptrit of Haut...

Apparatus and Rig used $y^{\prime}$, y

Time towine State of

fins. 
SOUNDING WIRE.

M. DOWN. UP. M. $2|32| 00|2| 4 \%$
FMS.

DREDGING CABLE.

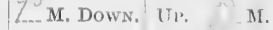

200

$\$ 300$

100

500

(i)

700

800

$9(0)$

1000

1100

1:00

1300

1400

1500

$16(0)$

1700

1800

1900

2000

2100

2200

$2: 300$

2400

2500

$\because 600$

2700

2800

2900

3000

3100

:3200

33300

3400

3500

$: 300$

$: 3700$

$: 300$

$: 3900$

1000

4100

.1200

4380

4400

thoo

4600

1700

1800

.1900 

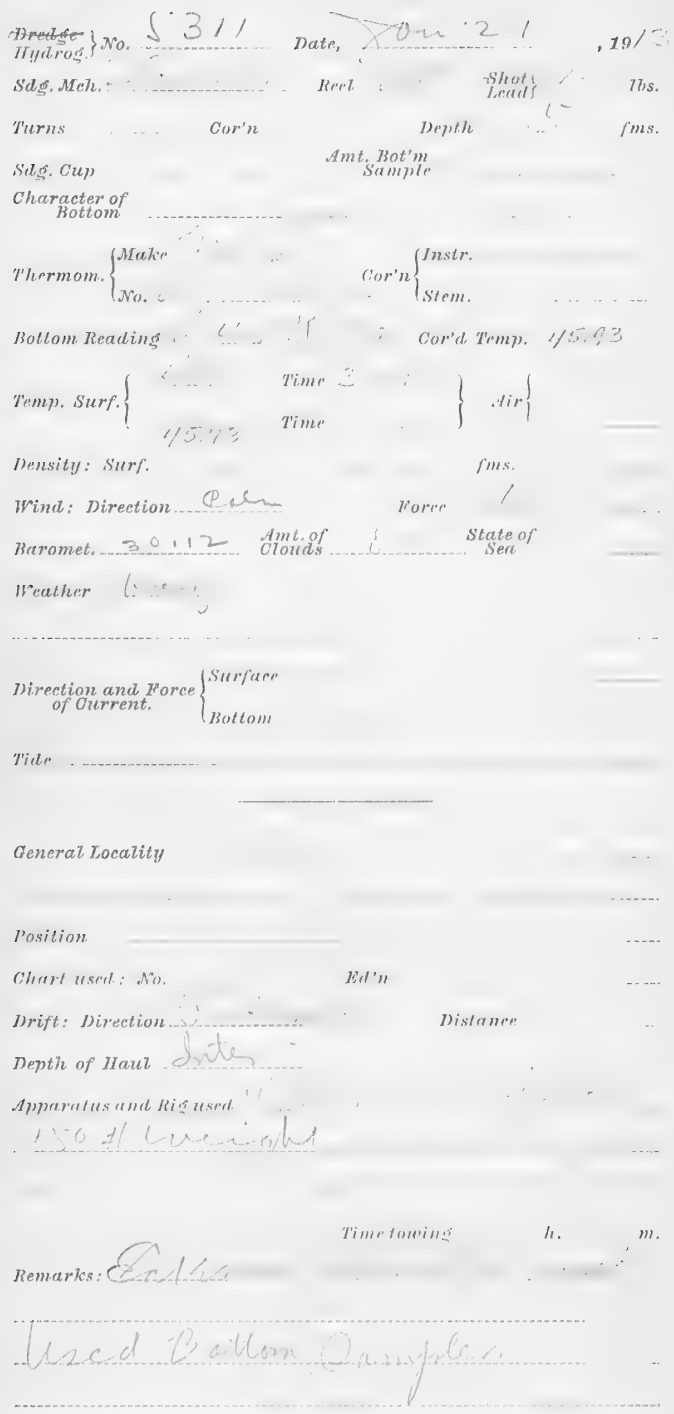
$11-1023$

SOUNDING WIRE.

U. Mown. Up, J M.

$215 \% 301365 / 3$.

FMS.

DIREDGING ('ABI,L.

1)

161)

200

300

400

500

(i)0

(1)0

800

()() ()

10010

1100

$1: 00$

1300

1400

1500

1600

1700

1800

1900

2000

2100

2200

$2: 300$

$\because 100$

2500

2600

$2 \pi 00$

2800

2900

$: 000$

3100

3200

$: 300$

$\$ 3100$

3,900

$: 300$

3700

3800

:3100

1000

1100

1:00

$4: 300$

4100

15,00

$4(6) 0$

.1700

4500

4900

M. Dowx. IV1. UE

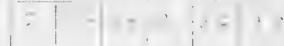

+

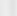


Dratgo
Hydrog No \& S J

"tl",

sils. Mrh.

tipl.

Shitols

lis.

Turns

Cor'n

Depth fins.

Sdg. Cup

Amt. Bot'm

Churacter of Bottom

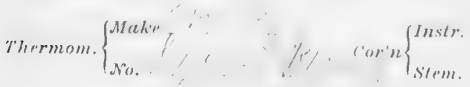

Bottom Reatinş.

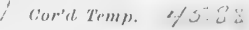

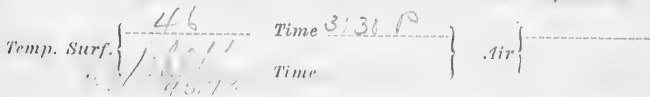
mensily: Surf: fins.

Wind: Direction. Foree

Hatromet, 26,12- Amt.of her.

lleulher

Direction and Force
of Current. $\left\{\begin{array}{l}\text { Surace } \\ \text { Botlom! }\end{array}\right.$

Tirle

General horetily

\section{I'osition}

Chart used: No. $\pi d ' n$

Irrift: Direction 1 Distumen

Depth of Hauz ... Jind

Apparatus and Rig used

10 i $1,0,0$ limmerks:

Timetureins h. $10 . .$.

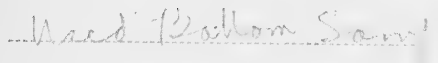




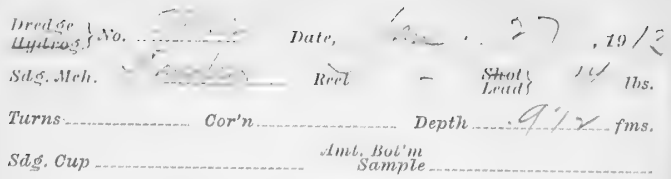
Character of
Bottom

Thermos. $\left\{\begin{array}{l}\text { Alate } \\ \text { No. }\end{array} \quad\right.$ Corn $\left\{\begin{array}{l}\text { Instr. } \\ \text { Stem. }\end{array}\right.$

Bottom Reading Cor'd Temp.

Temp. Surf. $\left\{\begin{array}{l}4 \\ 1 / 8\end{array}\right.$ $\left.\begin{array}{l}\text { Time } 10 \text { ied a } \\ \text { Time }\end{array}\right\} \operatorname{sir} 0^{\circ}$

Intensity: surf.

Hind: Direction

Baronet.? ?

Weather olein.

fms.

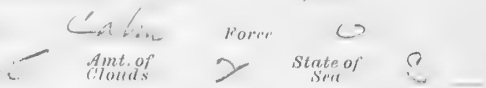

Direction and Force
of Current. $\left\{\begin{array}{l}\text { Surface } \\ \text { Bottom }\end{array}\right.$

rile

General Locality

Position

Chart used: No. $Z d i n$

Drift: Direction Distance

Depth of Haul .. of of

Ap)mentus and Misused

Time towing 7. $-m$

liemerlis:

- mice

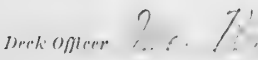

lieroriter. 


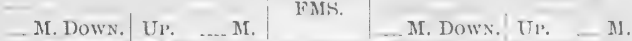
P.:

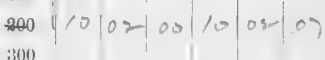

$: 3()$

400

500

(i)()

700

800

())()

1000

1100

$120(0$

1300

1.100

1,500

1600

1700

1800

1900

2000

$\approx 10$

2ะ200

:2:300

$\because 100$

2500

2600

$2 \pi 00$

2800

2900

3000

3100

$3: 00$

:3:300

3.100

3). 00

$: 3600$

$: 3700$

3800

3900

.1000

.1100

4:00

4300

4400

.1500

(1960)

$.17(1)$

.

4900 


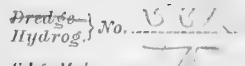

sillo...Hill.

Turns ffi..

Sit: $\{$. $(u)$

Chatrecter of

Bottom

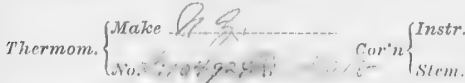

bitlom Reallins of

Gor't Timn. 1/, 68

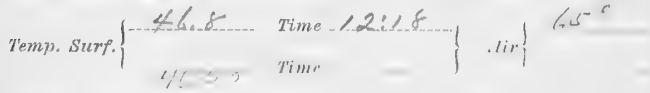

Mensily: surf.

fins.

Wind: Direction.. (

Force 0

lineromet. $30.1 \%$ Mme of 0

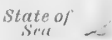

wrathrr $=$

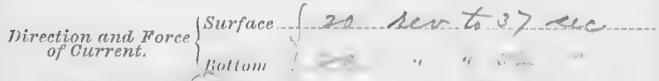
Titlo

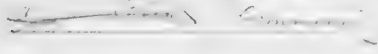

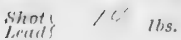
fins. Amt, Bot'm

Depth....

\section{Tin}

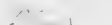

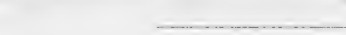

(iemeral loculily

Pnsition

Chart usel : No. .. Eil'n

Drift: Direction.. Cuncheled ... Distance

Deptr of Haut Dentia.

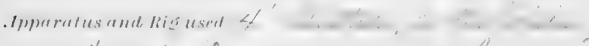

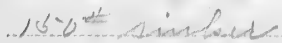

Time towing

7... $\bar{c} \ldots$

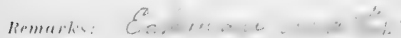

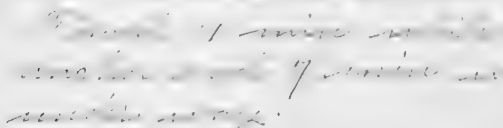


SOUNDING WIRE.

OM. Down. Ur. $Q_{\text {e M. }}$. $1217.00,122700$
Fas.

DREDGING CABLE.

DMr. Down. UP. D. M.

() $x: \because: 0 \quad=0400$

100

200

300

400

500

(i)

ro0

(⿻)

500

1000

1100

$12(0)$

1300

$1.4(00$

1500

1600

1700

1 Sitro

19010

$\because 000$

$\approx 100$

2000

:2:301)

$\because 100$

是(06)

$\because(i 00$

2700

Bsivt)

2!)

:3000)

$\$ 3100$

:1200

:3:300

:3100

3.200

:(6) (i)

:ละ)

:34)11

:3900)

4000)

1100

1901)

4:(3)

1100

1500

$f(600)$

1700

1800

(1000 


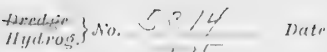
Sille 14.4

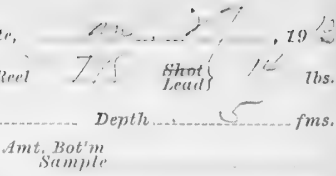

Turns Cor'n

Sil:. Cul Sampl

Character of Bollon.

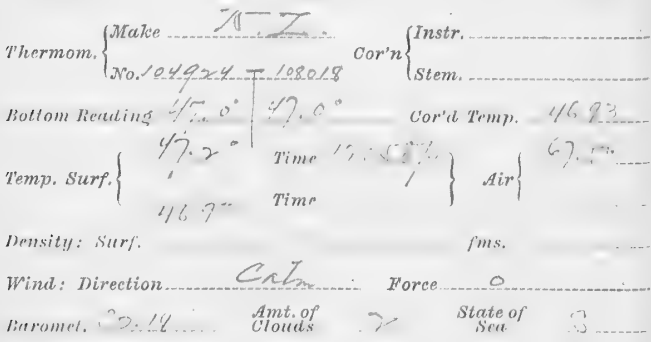
Wresther lie. Direction and Force
of Current. $\left\{\begin{array}{l}\text { Surface } \\ \text { Bottom }\end{array}\left\{\begin{array}{l}2, \\ 20\end{array}\right.\right.$ Tiale $\mathcal{F} / \mathrm{r}$,

General Locality

Position

Chart used: No Wit'n

Drift: Direction Aminaxal. Distance Denth of Haut Tnter

Apparatusand his uscut

Time towing 7., $22, m$

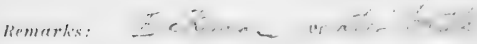

merkeoplentil.

Liecoriter

$11-10 z=3$ 
WMS

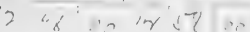

FMS. JM. Down.l UP. M.

()

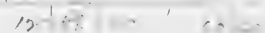

100

200

30)

$f(0)$

500

(i) (1)

7()

$\mathrm{s} 00$

90()

1000

1100

$1: 200$

1800

1400

1500

1600

1700

$1 \mathrm{~s} 00$

19)(

2000

$\approx 100$

$2 \div 00$

$23(30)$

$\because 400$

2500

$\because 600$

2700

asto()

:900)

$: 060$

3100

$\therefore \geq 0(1)$

:3ison

3100

3isto

:36)(

:รด0

उसi(0)

:900!

.10001

4100

fश:00

4300

$\$ 100$

.1500

4600

4700

4800

4900 
$\left.\begin{array}{l}\text { Hudase } \\ \text { Hydrog }\end{array}\right\}$ Wis.

Shle. Meh.

Turns Cor'n
Dats,

linel

Shont

Depth

Amt. Bot'm
Sample
.70 I

los.

fins.

Sds. Cup

Character of

liotion

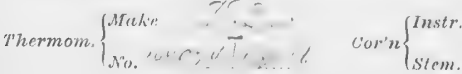

Bottom Reading $5 /{ }^{\circ}, U / 3^{\circ}$ Cor'd Temp...5L.08

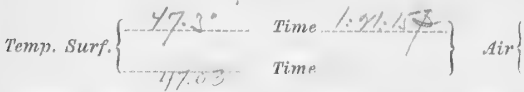

$(1)$

Density: Surf.

Wind: Hirertion Poren o

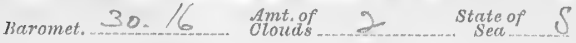

Weather

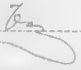

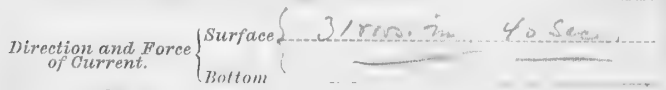
Tille... $\ \because \backslash$.

Generel Loectity

Position

Chart usarl: Aro.

Ed.'

Drift: Direction

frictines:

Distance

Depth of Ilaut Inites

Apparatus and hig used

Time towing

7 .

$m$.

Remorks:

Wire water dawe

$\therefore \lambda_{2} \hat{A} \ldots .$.

$1:$

I. 
29100

:8)(0)

3100

3200

:3is)

$: 3400$

3500)

:

37(0)

:3800)

:3100

f(0)0

4100

4.190

1300

1100

(5)

.1600

1700)

1800

4900 


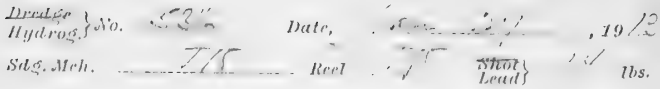

Turnis.

Cor'n

Depth fims.

Sid E. ('ul)

Amt. Bot'm
isampire

Character of

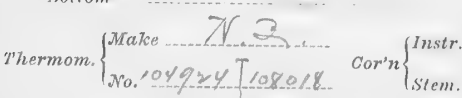

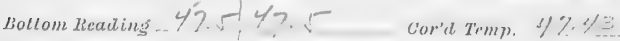

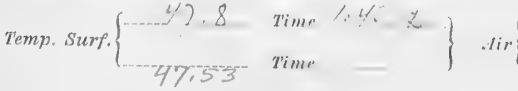

Density: Surf.

fms.

Wint: Direction.

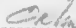

i.

Birromet. = , - Amt. of

Force 0

Weather 2i:3

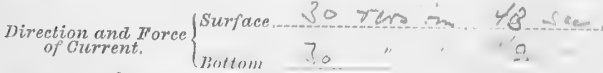
T.

Tiler $2:-1$

General Locality

Position.

Ghreld used: wh En'n

Drift: Direction Anctind. Distance...........

Depth of Haut rote.

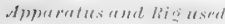

Timetowing th. If $m$

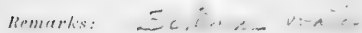


$11-1023$

SOUNDING WIRE.

M. Down. Uw. VM.

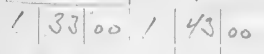

DREDGING GAIBLE.

FMS. $\vec{V}$ M. Down. Ur. V. M

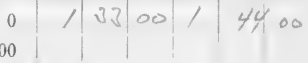

200

$: 300$

.100

500

(jo0)

\%00

800

900

1000

1100

1200

1300

1400

1500

1600

1700

1800

1900

3000

2100

2200

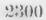

$29(10)$

$2 \% 00$

$\because 600$

$\approx 700$

$2 \mathrm{~s}(0)$

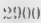

$\therefore: 000$

3100

:3200

33:300

i:1010

3.300

:3600

:70

: 3000

3900

.1000

.11010

$120 x$

1301

1.100

. 1500

$.160(0)$

1700

.1800

4900 


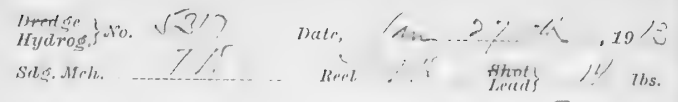
Turns _....- Cor'n
Depth. fims.

Sas. Cup

Character of

Botiom

Whermom. $\left\{\begin{array}{l}\text { Maker } \\ \text { No, } 1047 \times 4-108018\end{array}\right.$ Cor'n $\left\{\begin{array}{l}\text { Instr. } \\ \text { stem. }\end{array}\right.$

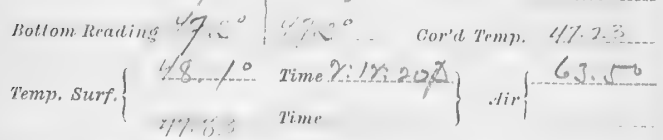

Density: Surf:

fims.
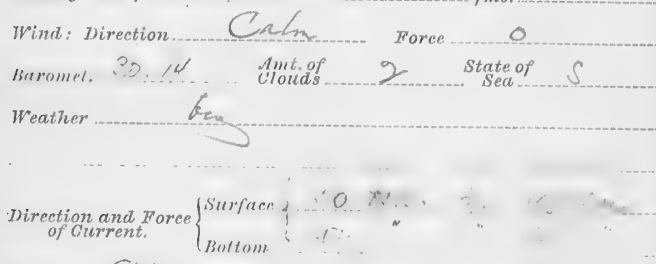

Tille $\cong$......

Amt. Bot'm
Sample

_.....

General Lorrtity

\section{Position.}

Bhart used: tro. Hed'll

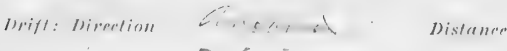

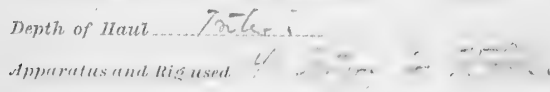

Time towing -

th... $/ \ell$.

Remurks: $=,-, \ldots \ldots+\cdots, \ldots$

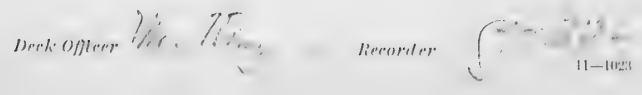


11-1023

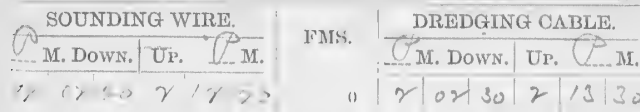

100

200

:300

.1100

inot)

(i)i)

700

Sit)

(1)()

1000

1100

12)( )

1:3(x)

1.100)

1500

$16(t) 0$

1700

1500

19)(3)

?0(1)

2100

2200

21300

$\because 100$

250()

$\therefore(i)()$

$\because 2(1)$

$: 80()$

:3!)u(t)

:I)(H)

:3100)

:1:2)(1)

:3:)(0)

:3101)

:isio 00

:(i)

:3)(1)

:3:(1)

:新)

I(1) 0

4100

1?(1)

1:300

1100

fis ()

Ifi()

1700

1500

1900 


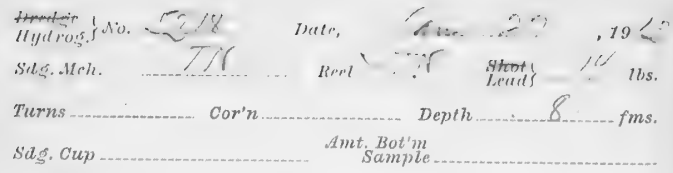

\section{Character of} kottom

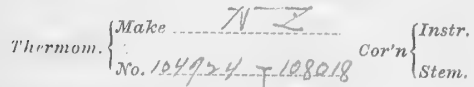

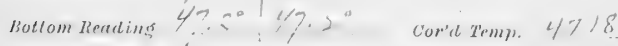

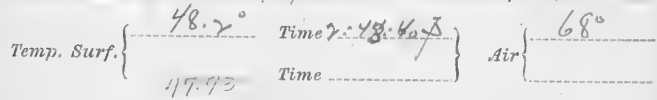

Density: Surf.

fims.

Wind: Direction... Parce 0

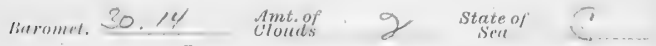

Weather .......

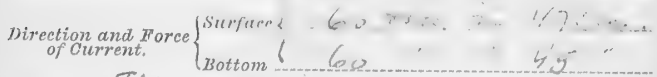

Tilte $7 / 1$

\section{General Locality}

\section{position}

chart used: No. $\pi d^{\prime} n$

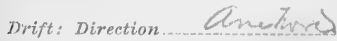

Distance

Depth of Haul... Trete.

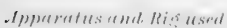

Time towing h. $/ / m$

Remarks:

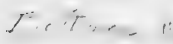

$\checkmark 7$. ( 
$11-1023$

SOUNDING WIRE.

M. Down. UP. C.. M. $v \longdiv { 3 3 4 } 2 4 3 4 0$
FMS.

DREDGING CABLA.

M. Down, lil. D.

1)

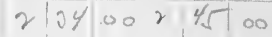

100

200

$: 30)$

4110

500

(60)

ז00

800

900

1000

1100

1200

1300

1400

15001

$16(0)$

1700

1800

1900

2000

2100

2200

2300

$\because 400$

2500

2600

$2 \pi 00$

2800

2900

$: 3000$

$\$ 100$

$: 300$

:3300

:3100

3500

:

$3 \% 00$

3800

$\$ 00$

1000

$\$ 100$

4200

4300

.1100

4500

4600

4700

1500

4(901) 


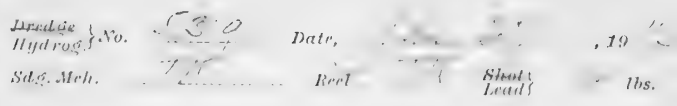

Turns

Cor'n

Depthi

.. fims

Sdg. Oup

Amt. Bot'm

Chroracter of

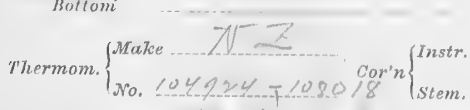

Rollom Readins

cor't Temp. 47.78

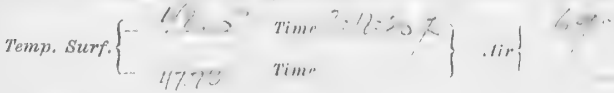

7)ensity: Surf:

fins.

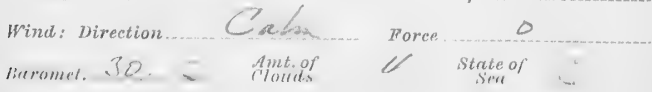

H'inther

Direction and Force
of Current. $\left\{\begin{array}{l}\text { Surfuce } \\ \text { Diollom, }\end{array}\right.$

ridi. s...'

(inllerel Lorrlity

I'osition.

Chart used: No. $I l d ' n$

Drift: Direction..... Clstchenes).

Distance

Depth of Haul $\sqrt{x_{2}}$

Ipparatus and Risutesed

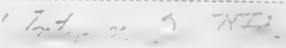

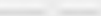

Timelouint h. :...

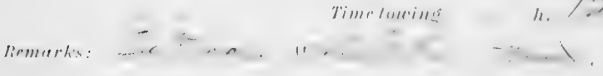

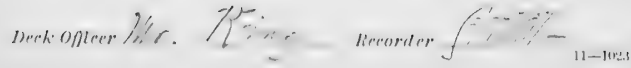


SOUNDING WIRE.

Ar. Down. UR. Mr.

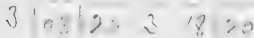

DREDGING CABLA.

FMIS.

I M. Dow. UR. (N).

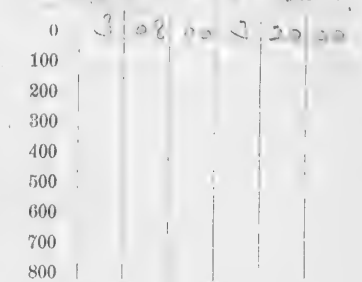

900

1000

1100

1200

1300

1400

1500

1600

1700

$-1800$

1900

2000

2100

2200

2300

2400

2500

2000

2700

2800

2900

3000

3100

3200

3300

3400

3500

3600

3700

3800

3900

4000

4100

4200

4300

4400

4500

4600

4700

4800

4900 


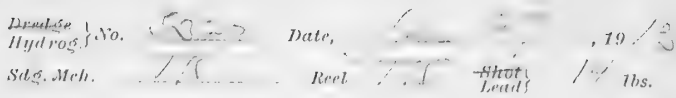

\section{Turns}

Cor'n.

Depti

fins.

Sdg, Cup

Amt. Bot'm

Character of

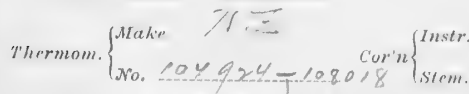

Bottom Reading

Temp. Surf. $\{$.

$\therefore$

Corre Trmp. 45.33

Jensity: Surf.

$$
10,7=-3
$$

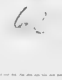

Wind; Direction

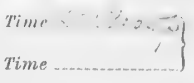

Air

fins.

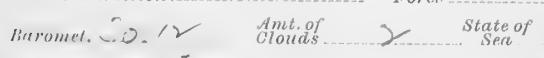

Irealler

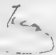
Sert

ing.....

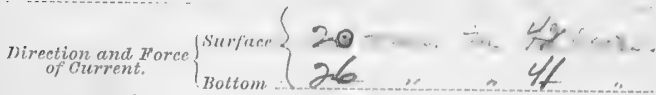

Tide

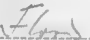

(imaral boralily

position

Chart used: No, Ed'n

Drift: Direction

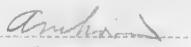

Distance.

$-$

Depth of Haul

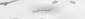

Apperrolus and Riş Usint

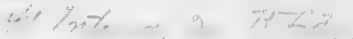

Time towing

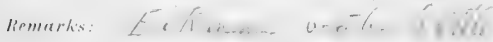

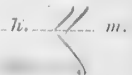
neret onierer
lir. yizis.

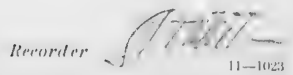


SOUNDING WIRE.

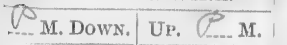

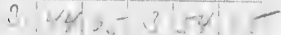

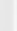




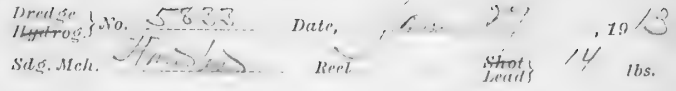
Turns
Cor'u
$D(p) t h$
fins.

Sel.s. Cup

Amt Rot'm

Character of

Thermom. $\left\{\begin{array}{l}\text { Make .... } \\ \text { No. }\end{array}\right.$ (orn $\left\{\begin{array}{l}\text { Instr. } \\ \text { Stem. }\end{array}\right.$

Bottom Reading Cor'd Temp.

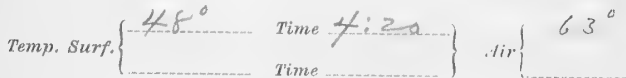

Density: Szrrf. fins.

Find: Direction

Force ... 0

Birromet. 33.. Amt.of

0 State of

Weather

Whether

1)irection and Force
of Current. $\left\{\begin{array}{l}\text { Surface } \\ \text { Buttom }\end{array}\right.$

Tider

General Locatity

Position

Chart used: No.

$l d^{\prime} n$

Difit: Direction

Distance

Depth of Haul ... toffore

dpporatusand Rim used

Time lowill

h.

$m$.

Remnrkes: 
SOUNDING WIRE.

.... M. Down. UP. M.

00

200

300

400

500

600

700

800

900

1000

1100

1200

1800

1400

1500

1600

1700

1800

1900

2000

2100

2200

2300

2400

2500

2600

2700

2800

2900

3000

3100

3200

3900

3400

3500

3600

3700

3800

3900

4000

4100

4200

4300

4400

4500

4600

4700

4800

4900
DREDGING CABLE.

AM. Down. Ur. M.

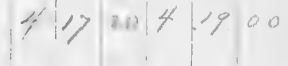

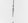

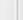

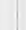




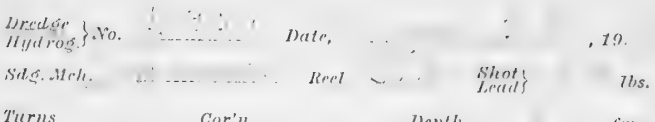

Turns Cor'n lepth lims.

Sids. $(u)$ )

ImI Bot'm!'

Character of Boltom

Thermom. $\left\{\begin{array}{l}\text { Make I } \\ \text { No. }\end{array}\right.$

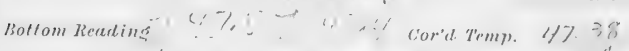

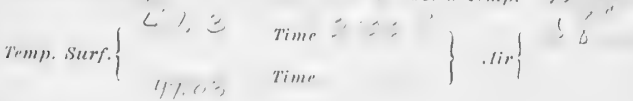

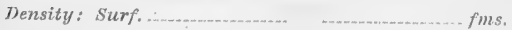

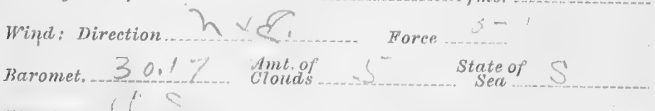

IIeather

Direction and Force
of Current. $\left\{\begin{array}{l}\text { Surfarr ? } \\ \text { liottom }\end{array}\right.$

Tili"

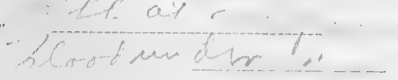

Gienirerl bocalit!

\section{Insition}

Chart used: No. $x d$ 'n

Drift: Direction Und oved..... Distance

Depth of Haut ....shite.

Apparatus and Rig used

$$
L^{\prime} \int_{1}+T_{0} \rightarrow T_{0} \times
$$
I

\section{Timetorrints}

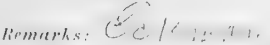

(imerinitis:

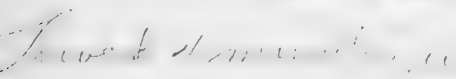

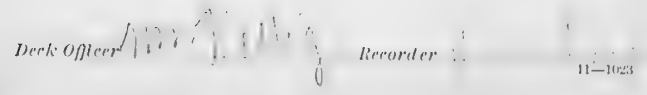


SUUNDING WIRE.

M. I)own.| U1. .... MI.

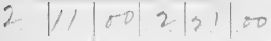

FNS.

DREDGING CABLE.

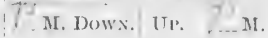

.

0

10)

200

:34) 0

.100

500

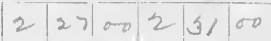

(io)

700

800

90

1000

1100

$1: 00$

1300

1400

1500

$160)$

$1 \% 00$

1800

1900

2000

2100

2200

$2: 300$

2400

2500

$\because 000$

2700

21500

2900

$\$ 3000$

$\$ 100$

$3: 200$

3300

3100

3500

$\$ 300$

$: \% 00$

$\$ 3800$

$: 3900$

1000

4100

1200

$4: 300$

4100

4500

4600

1700

4800

.9000 


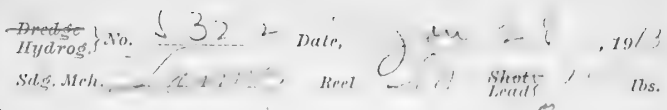

\section{Turns}

Cor'n

Depti

8 fins.

Sil is. Cup

Amt, Bot'm

Character of inotiom.

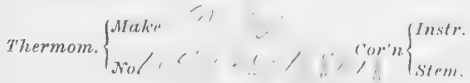

Bottom Reading $0^{\prime},+$, , , Cur'd Trmp. 47.53

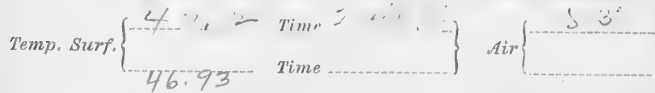

Density: Surf. fins.

Wind: Direction...h ostele Foree $0-1$

Baromet. $3,0,18$ Amt. of Single of Weather C is

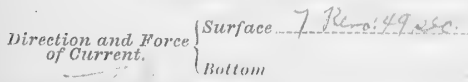

ricls

(imenrel horctilt)

Pesilion

(ihert usad: .1) EN'n

mrifl: Divertions i, o ,.. !

I)

Apparatus and kig used \&f ' ' Csioff bue ingtat

Time towins 7.. $)(1, m$.

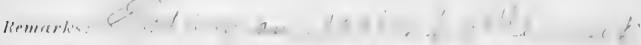

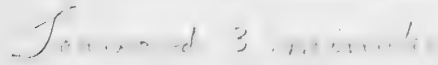

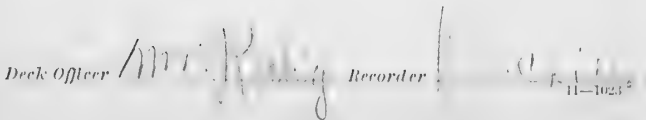


SOUNDING IVIIRE.

J. Down. Ur. M. $2.391: 2=8$
FATS DIREDGING (TABTLE

I. M. Dow. UT. i.... M.

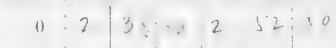

200

300

400

500

(50)

700

800

900

1000

1100

1200

1300

1.100

1500

1600

1700

1800

1900

2000

2100

$2: 00$

$2: 300$

$2: 100$

2500

2600

2700

2500

2900

3000

$\$ 3100$

:300

:300

$: 3100$

3500

$: 600$

ละช 00

3800

3900

.1000

4100

f 200

1:300

4.100

4500

4600

4700

1800

.1900 


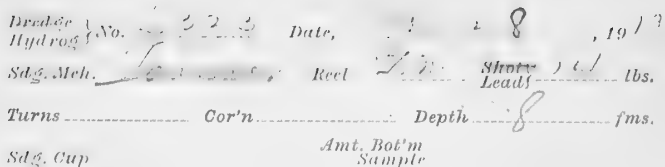

Sists. (iup)

Sacuind

Choriecter of

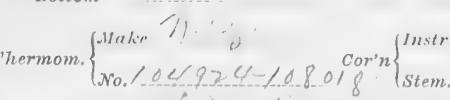

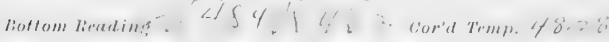

Temp. Surf. $\left.\left\{\begin{array}{l}\therefore, \text { Tim. } \\ \text { Time }\end{array}\right\} \operatorname{Air}\right\}$

Density: Surf. fims.

Wind: Direction..n ath porce $0-1$

Baromet. 30.1 S Ant of 7 . State of 5

Iitallere o, is as

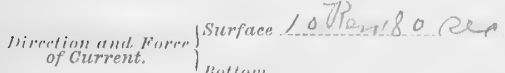

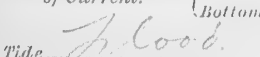

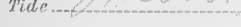

Gimerat Lorealit!

\section{position}

Chart used: No, Drift: Direction

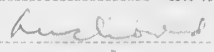
Distance Depth of Haut Apparatus and Rig used $4)_{2} C_{3}$ 1 $i_{i}$

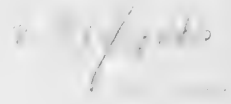

Time-lurrin.

hemurh: é

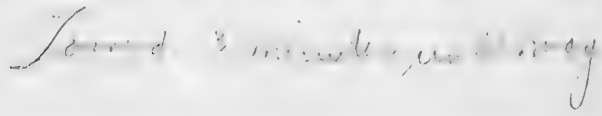


SOUNDING WIRE.

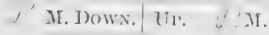

$3|02| 30: 3|12| 30 \mid$
FMS.

DREDGING CABLE.

I...M. Down, U1. . 11.

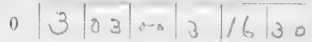

100

200

:3(0)

10k

50)

(i) (1)

rou

B(1)

900

1000

1100

1200

1300

1.100

1500

1600

1700

1800

1900

2000

2100

2200

2300

2400

2500

2000

2700

2800

2000

3000

3100

3200

:3:itin

3400

3500

3600

3700

3800

:3900

4000

4100

f.

4300

4100

4500

4600

fivi

4800

4000 


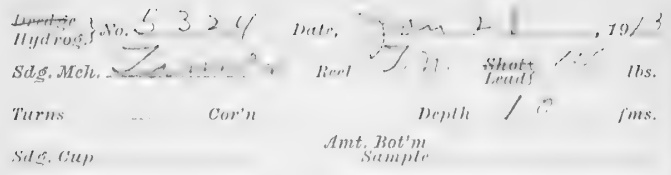

Character of
Bollonil

sitilil.

Thermom. $\left\{\begin{array}{l}\text { irakin } \\ \text { No. } 104924-108018\end{array}\right.$

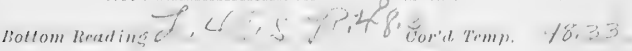

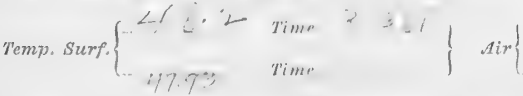

nensityl: Surf:

Wind: Direction...n the

lixtrumet. $3(1,1$ ! Alowicus ?

fims.

wealler. 7, 15

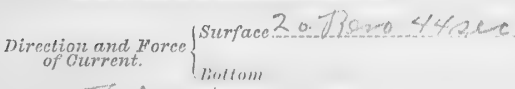
Tiue of Cord

Generert Lomulit!

Position

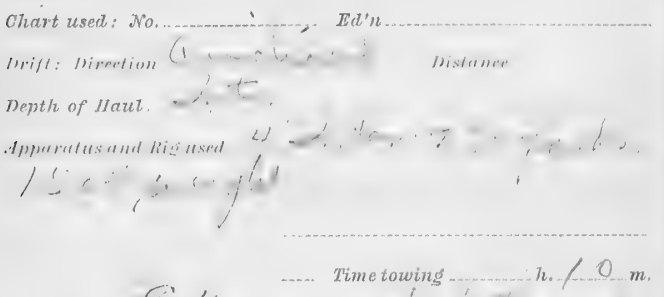

Remerk: (E)

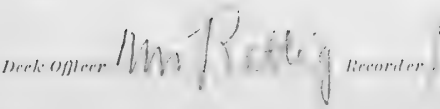


$11-1023$

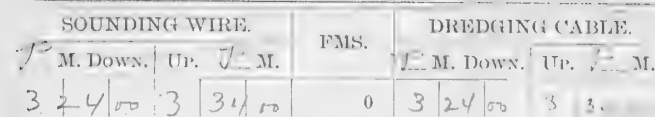

$3240: 3 \mid 31 / 10$

10()

200

:30)

(1)0

i() ()

(i)

700

$8(1)$

()00

1000

110()

1200

130)

1.100

1500

1600

1700

18(0)()

1!)(1)

30()()

¿1( )

2)-3(1)

2:101)

$\because 1(1) 1$

$\because$ i) (1)

ati(x)

$27(1)$

$\because \times 10)$

-3)(1) ()

:3)(u)

3100

$\therefore 300$

:isiono

:31(u)

$: 3)^{2}(0)$

:i(jik)

:3)( )

:सun

:3:30)

.100)

1100)

.1?(1)

I:B(1)

.1160

1.iv)

Ifici)

17(1)

1800

4000 


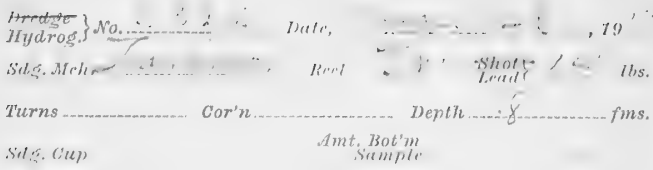

Character of

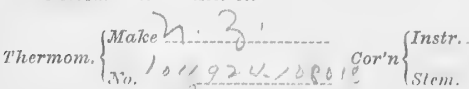

Bollom lieredins.

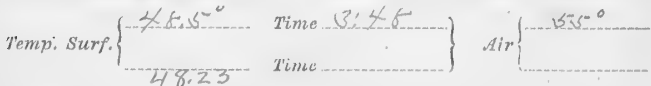

llensity: Surf. fms.

Wind: Direction. Qandabe Force o - )

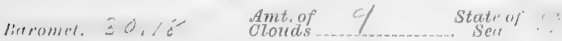

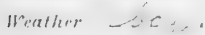
Direction and Force
of Current. Rertlom

Tide

$$
\rightarrow \text { lintlam }
$$

$\therefore$

\section{General Loralily}

\section{I'esition}

Chavt used: No. $x d ' n$

Drift: Direction Ciucl Distance. Depth of Haut. Lute

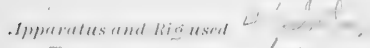
t5on cuer.int

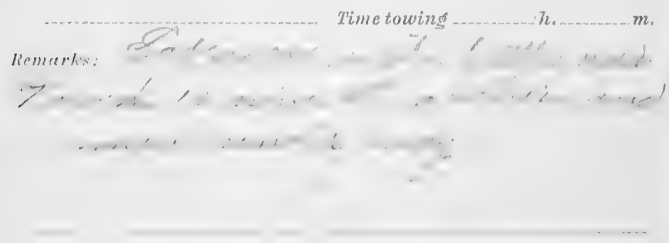




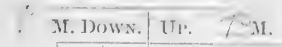

FMS.

M DOWN TI. Nis

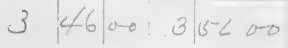

()

100

200

300)

(10)

500

(i)o

ז00

800

90

1000

1100

1200

1300

1.100

1500

1600

$1 \% 00$

1 sison

1900

$\because(900)$

:100

מำ

2:300

?100

25010

2600)

$2 \%(0)$

ख्या10

?!)(1)

:3000

$\$ 100$

:3)

(i):(נ)

:3100

i3ivo

:(3)

:3ino

:3ง

:300

10011

.1100

$13(x)$

fis $3(x)$

$f(1)$

fist)(1)

f(i)e

1701

(1:i0)

.1900 


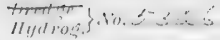

Sils. $11+h$.

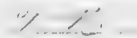

Turn:

Cor'n

Sirls. ('u)

Character of

Burllom

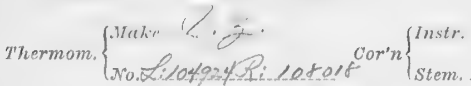

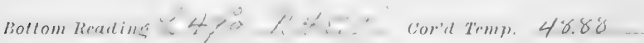

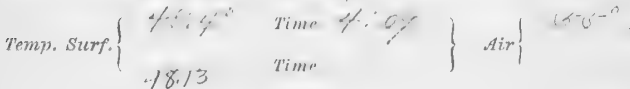

$$
.18 .13
$$

1)ensity: Sturp. fins,

Wind: Direction. Priabe

Baromet. $30,19 \quad$ Amt. of Force.

weather flezpl

Direction and Force
of Current.

Tide.

\section{General Loratily}

\section{Position}

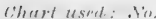

E'l'

Drift: Direction Distance

Deptr of Haur duster.

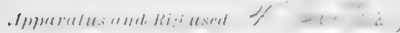

$1: 0,2.2 .1$.

Time towing

$7.10 \ldots$

liemurkes:

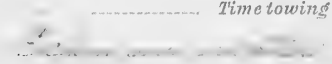

- 
11-1023

SOUNDING WIRE.

W. M. Dows. Ur. QR.

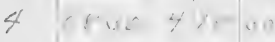

Fas.

100

200

300

$\$ 00$

500

600

(10)

×00

900

1000

1100)

1200

1800

1.400

1500

1600

1700

1800

1900

2000

2100

2200

2:3)

$\because 100$

$\because 2003$

$\therefore(60)$

$2700)$

3900

: 2900

$: 000$

3100

:3:(0)

(3):(1)

:314)

:"พ)(11)

;난)

(ล)

:सพ111

:3901

40100

f(10)

f:(100)

(1:3)

$\ln 100$

.

4600

1700

4800

1900
DREDGING CABLE.

PM. Down. UP. P. M.

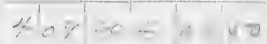

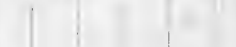




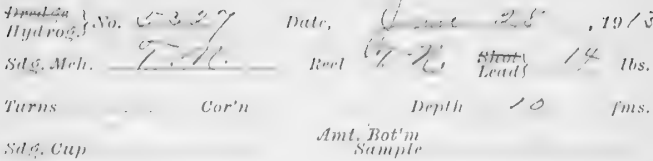

Character of

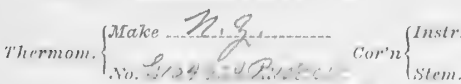

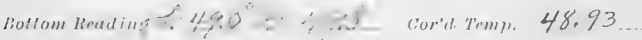

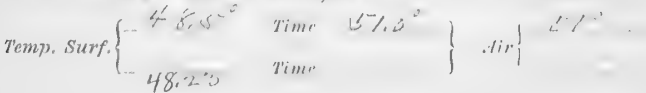

7) cusity: Sturf

fms.

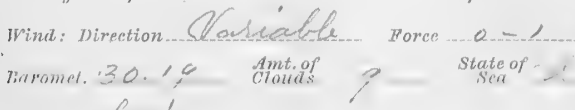

Weather finget.

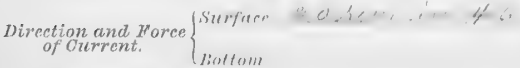

Tille

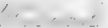

Geurret Lomlit?

Pesilion

Chart used: No. End'n.

Drift: Direction....QReucbinareaL ...., Distance

Depth of Haut . strester.

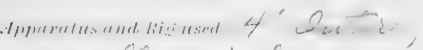

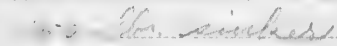

Time towing h. $10 \ldots . . m$.

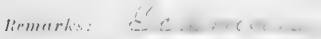

$-1 \ldots x^{-1}$

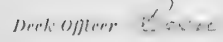


SOUNDING WHE G M. Hown. Ir. It M.

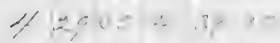
IMS.

DIREDTINA ('ATSLE.

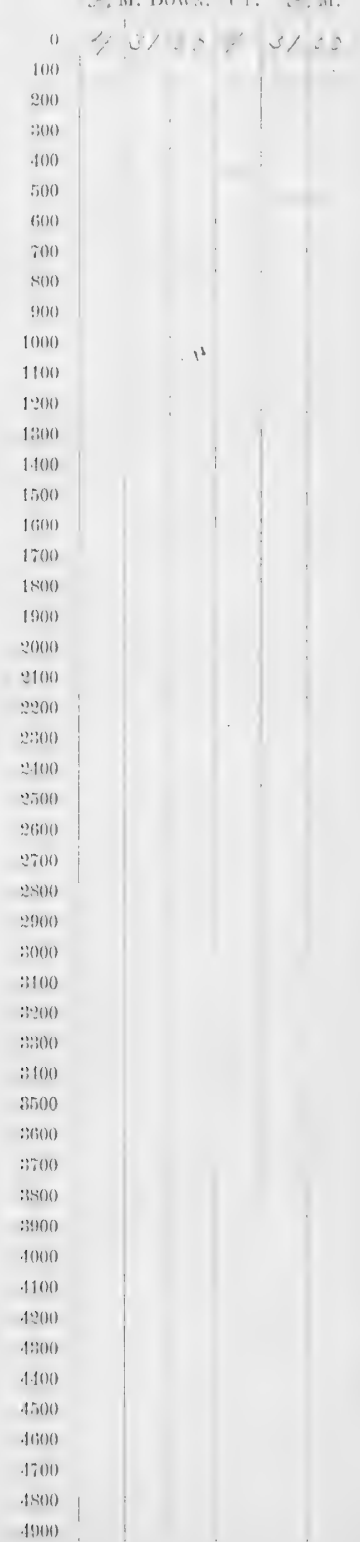




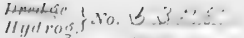

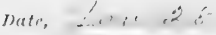
, 1013
Sids...Heh.
Y $1, \ldots . .$.

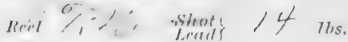

Turns

Cor'n

Depth:.... $/ L$ frms.

Sals. (iu $)$

Amt, $B$ sot'm

Chrirater of

Bottom

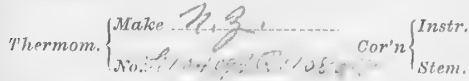

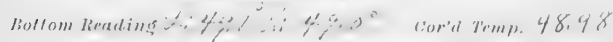

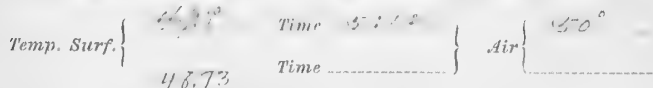

Density: Surf.

fms

H"ind: Hirnetion

Forrw

liaromet. ? , Mmet of

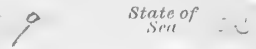

Weather

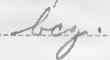

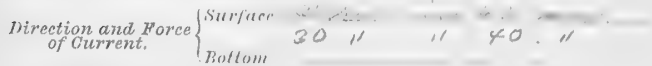
Tite...

(inneret borulit!

\section{I'osilion}

Chart used: No. $\xi \ell^{\prime} n$

Hrift: Disreliul

lisfunes

Depth of Haut $>$ thate.

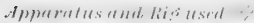

< $50^{\prime \prime}, 1, \ldots+\cdots$

Till, tourin.

11. ‘ 0 :

$\| \cdot m+1 \cdot l_{2}$.

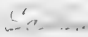


SOUNDINA WIRE

DM. Down! Ur. PM. $510000: 51000$
Tist

DREDGING CABLE.

QM. Down. U1. PM.

1)

I1)(1)

$20(1)$

$::(3)$

1100

500

(ii)

(0)

$S(1)$

()แอ

(1)()(

11011

1:2()!)

$1: 0()$

1.100

1500

$16(1)$

1700

1800

19(1)

$2(x)()$

$210 k$

$20(0)$

$\because 3(1)$

2900

$25(10)$

2000

?าม10

$\because 400$

2900

$: 2000$

3100

:?:(1)

(3:)

:i100

$\sin 00$

:3(i)1)

:ะสา

$: \$ 400$

$: 900$

.1000

1100

4200

.1:301

1400

Ans(x)

Asio)

1700

4800

1900

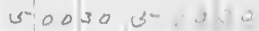




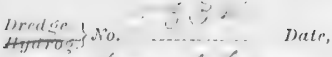

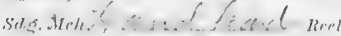

Silrot $1 \dot{4}$ los.

Turus

Cor'n

l) $m / h$

fins.

Sils. Cup

Amt, Bot'm

character of

Thermom. $\left\{\begin{array}{l}\text { Malie } \\ \mathcal{N} o .\end{array}\right.$ Cor'n $\left\{\begin{array}{l}\text { Instr. } \\ \text { stem. }\end{array}\right.$

liotlom Reretills Cird Trmp.

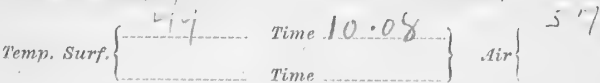

Density: Surf.

fims.

Wind: Direction.

$0 \frac{6}{6}$

liaromet.

Ant.of , h state of

Heather

Direction and Force $\left\{\begin{array}{l}\text { Surfirm } \\ \text { of Ourrent. } \\ \text { Bottom }\end{array}\right.$

Tille

Vimprul homtily

Pasition

Chart used: No. Lit'n

Drift: Direction Distance

Deptiz of Haul

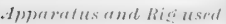

Timelorists

h.

III

liemurlis: 
SOI:NI)IN(: WIIRE.

M. I)(MN. II). . MI.
1)TREDYING ('AIBTLE.

TML. A Mr. Dows. Tr. Af .I.

$0|10| 06|40100.7|^{50}$ III)

2010

$: 2())$

10)

i) $(0)$

(i) ()

;()(1)

8()()

())(1)

1(1)(10)

1100

1?0)

1300

1.100

150)

I (i) 0

$1 \% 00$

1800

$1901)$

?(3)

2100

13) 0 ()

1):3()()

$\approx 100$

3500

$\therefore(0) 0$

$27(k)$

खsion

9900

$: 3000$

$\$ 3100$

$2: 300$

:3:?00)

:310)

3.000

$\because 600$

:3in (1)

$: 3400$

$: 3900$

.10(0)

1100

$f^{2}(0)$

(i:)(i) ()

4100

1. $50(0)$

sicion

.1700

14800)

.19)( 
Mral se l No it: ? :

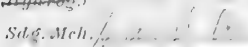

Turns

Cor'n

Sid es. (tu)

Ame. Bot'm

Chireracter oy

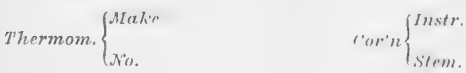

Bottom Reading Cor'd Temp.

Temp. Surf. $\{$. Time $\{0,4(0), 5]$

Density: Surf.

Wind: Direction...5). fins:

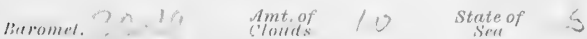

\section{INealler}

Direction and Force $\left\{\begin{array}{l}\text { Suras } \\ \text { of Current. } \\ \text { Bottom }\end{array}\right.$

Ti,l.,

(ionerul Loratily

l'esition

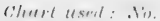

Drift: Direction Distance Depth of Haut.

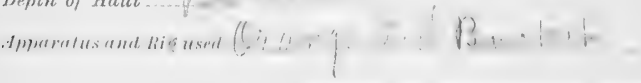
$m$. liminurh: 


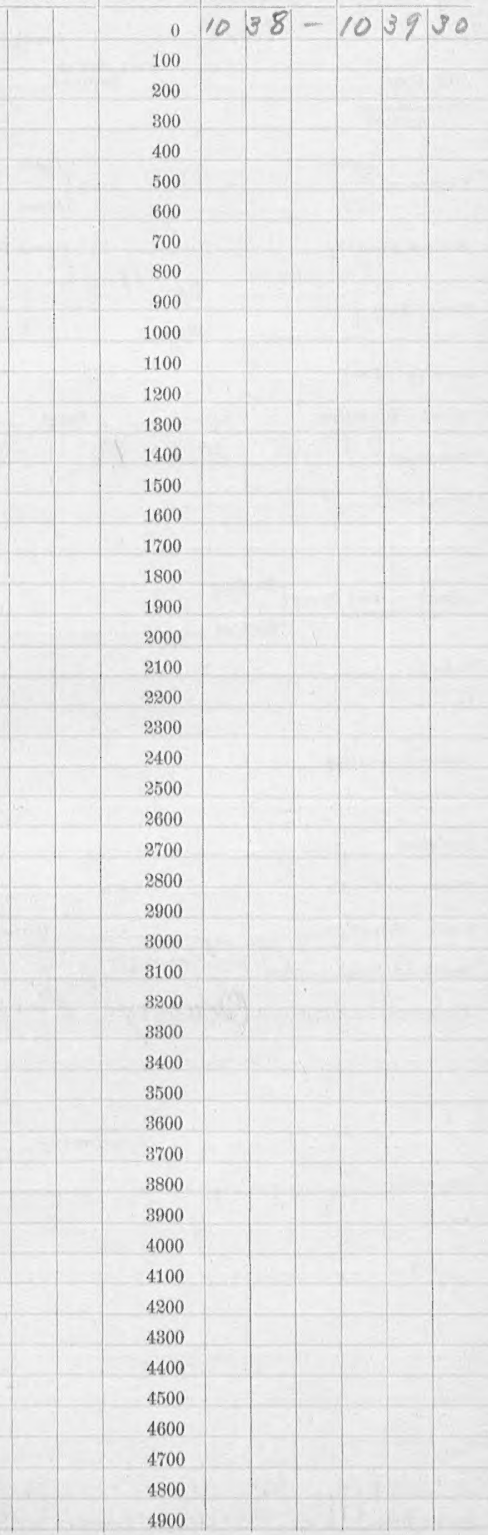




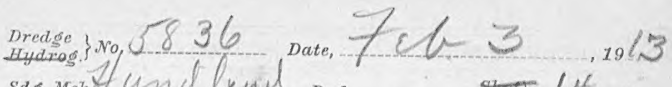

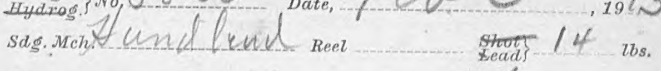

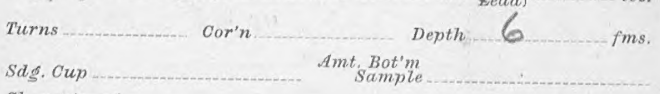
Character of
Bottom

Thermal: $\left\{\begin{array}{l}\text { Make } \\ \text { No. }\end{array}\right.$

$$
\text { Corn }\left\{\begin{array}{l}
\text { Instr. } \\
\text { Stem. }
\end{array}\right.
$$

Bottom Reading

Cord Temp

Temp. Surf. $\left\{\begin{array}{ll}144 & \text { Time } 11.06 \\ \text { Time }\end{array}\right\} \operatorname{sir}\left\{\begin{array}{l}48 \\ \text { Tim }\end{array}\right.$

Density: Surf.

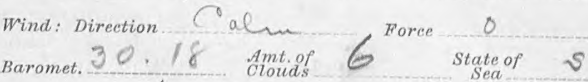
weather oc 7

Direction and Force
of Current. $\left\{\begin{array}{l}\text { Surface } \\ \text { Bottom }\end{array}\right.$

Tide

General Locality

Position

Chart used: No.

Eden

Drift: Direction

Distance

Depth of Haul .... 5 Clive

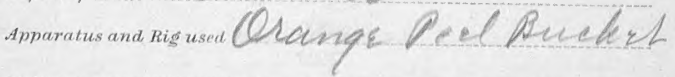

Remarks:

Time towing _....... $h$. $h$.

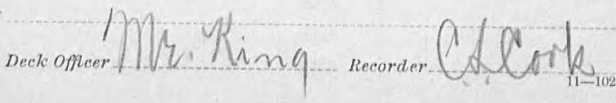


SOUNDING WIRE.

M. DOWN. UP. _... M.
FMS.

DREDGING CABLE.

0

A.M. Down. UP. A M.

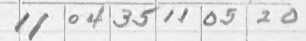

100

200

300

400

500

600

700

800

900

1000

1100

1200

1300

1400

1500

1600

1700

1800

1900

2000

2100

2200

2300

2400

2500

2600

2700

2800

2900

3000

3100

3200

3300

3400

8500

3600

3700

3800

3900

4000

4100

4200

4300

4400

4500

4600

4700

4800 


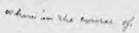

81049247700818

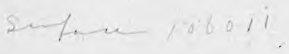

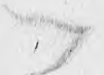

